\title{
Thermal Effects on the Nonlinear Forced Responses of Hinged-Clamped Beam with Multimodal interaction
}

\author{
A. N. Ndoukouo a,1, ${ }^{*}$, G. S. M. Ngueuteu ${ }^{b, 2}$ \\ ${ }^{a}$ Department of Architecture and Engineering Arts, Fine Arts Institute, University of Dschang, P.O Box 31, Foumban, \\ Cameroon \\ ${ }^{\mathrm{b}}$ Independent Researcher, P.O. Box 55, Ntui, Cameroon \\ 1 ahoudoungamie@aims.ac.tz; ${ }^{2}$ ngueut@yahoo.fr \\ * Corresponding Author
}

\section{ARTICLE INFO}

Article history

Received 04 August 2021

Revised 16 August 2021

Accepted 18 August 2021

Keywords

Thermal loadings;

Nonlinear vibrations;

Multimodal interactions;

Internal resonance;

Frequency response curves

\section{ABSTRACT}

Nonlinear analysis of a forced geometrically nonlinear HingedClamped beam involving three modes interactions with internal resonance and submitted to thermal and mechanical loadings is investigated. Based on the extended Hamilton's principle, the PDEs governing the thermoelastic vibration of planar motion is derived. Galerkin's orthogonalization method is used to reduce the governing PDEs of motion into a set of nonlinear non-autonomous ordinary differential equations. The system is solved for the three modes involved by the use of the multiple scales method for amplitudes and phases. For steady states responses, the Newton-Raphson shooting technique is used to solve the three systems of six parametric nonlinear algebraic equations obtained. Results are presented in terms of influences of temperature variations on the response amplitudes of different substructures when each of the modes is excited. It is observed for all substructures and independent of the mode excited a shift within the frequency axis of the temperature influenced amplitude response curves on either side of the temperature free-response curve. Moreover, it is found that thermal loads diversely influence the interacting substructures. Depending on the directly excited mode, higher oscillation amplitudes are found in some substructures under negative temperature difference, while it is observed in others under positive temperature change and in some others for temperature free-response curves.

This is an open-access article under the CC-BY-SA license.

\section{Introduction}

It has widely been shown that a beam with dissymmetric boundary conditions, precisely a Hinged-Clamped beam, presents a rich dynamic due to interaction between its substructures [1]-[9]. If one takes the first three substructures of a nonlinear Hinged-Clamped beam, it can be seen that their frequencies are naturally commensurable. The second mode frequency is three times the frequency of the first mode : $\omega_{2}=3 \omega_{1}$. The frequency of the third mode is given by a linear combination of the frequencies of the first and the second mode, namely : 
$\omega_{3}=\omega_{1}+2 \omega_{2}$. As a consequence, there is an internal resonance resulting from energy transfer between these substructures [5-7]. Lee et al. [7] have shown, for example, that if the second mode is excited, it is possible to have the non-excited first mode responding with a larger amplitude.

To the best of our knowledge, minimal studies are available on the influence of temperature change on the substructures of a Hinged-clamped beam involving internal resonance, whereas several studies have shown that a moderate temperature change can be of great influence on structural responses [10]-[12]. Xia et al. [12] stated that changes in the vibration characteristics of the structure due to damage might be smaller than changes in ones due to variations in temperature. Moreover, it is widely known that structures are permanently exposed to the thermal field from accidental or environmental origins that induce temperature variation of their members [10]-[24]. Yaobing et al. [13] have paid attention to the temperature effects on the nonlinear vibration behavior of Euler-Bernoulli beams with different boundary conditions. Effects of uniform temperature rise on the structural responses have been investigated through the authors limited their study to singlemode analysis for the sake of simplicity. They showed that the enhancement of the constraint conditions from Hinged-Hinged, Hinged-Fixed to Fixed-Fixed reduces the temperature effects on the vibration behaviors and that the hardening behavior tends to increase for positive temperature change and to decrease for a negative one. Warminska et al. [16] studied the nonlinear dynamics of a reduced multimodal Timoshenko beam subjected to thermal and mechanical loadings. They considered the first three modes of a simply supported beam at both ends and investigated the influence of temperature on primary resonances around the frequencies. Meanwhile, several studies [25]-[29] have shown that although there are commensurable linear natural frequencies such as $\omega_{3}=\omega_{1}+2 \omega_{2}$ (where $\omega_{n}=n^{2} \pi^{2}$ ) there is no internal resonance in the case of Hinged-Hinged beam because of the vanishing of the coupling coefficients leading to internal resonance, unlike the cases of Hinged-Clamped and Clamped-Clamped.

The research contribution is to deal with the nonlinear analysis of a forced geometrically nonlinear Hinged-Clamped beam with three modes interactions and submitted to thermal and mechanical loadings. The goal is to investigate the effect of temperature change on the steadystate responses of different modes when the excitation frequency is near the frequency of a given mode. It is observed for all substructures and independent of the mode excited a shift within the frequency axis of the temperature influenced amplitude response curves on either side of the temperature free-response curve. The shift depends on the magnitude and sign of the temperature difference. Oscillation amplitudes of the substructures are remarkably influenced by thermal effects and diversely respond to temperature change depending on the directly excited mode, the sign, and magnitude of temperature difference. For some substructures, higher oscillation amplitudes are observed under negative temperature difference, while it is observed for others in the presence of positive temperature change and for temperature free-response curves for some others.

The structure of the paper is organized as follows: In section 2, the governing equations of the beam for thermoelastic vibration are derived using the extended Hamilton's principle. The PDE of the planar motion is reduced to a coupled nonlinear ordinary differential equations by means of modal projection and then by using the Galerkin orthogonalization method. The system is solved for amplitudes and phases of the three modes using the multiple scales method. The steady-state responses are numerically obtained for different temperature changes in section 3. Some numerical results and discussions are presented in section 4 to illustrate the influences of temperature variations on the response amplitude of the substructures when a given mode is excited. Some concluding remarks are drawn in section 5. 


\section{Mathematical formulation of the thermoelastic problem}

Consider a slender, initially straight hinged-clamped beam shown in Fig. 1. In this study, $u$ and $w$ denote the axial and transverse deflections of the beam, respectively. $x$ is the distance along the undeflected beam, $t$ is the time, and $l$ is the beam length. $m=\rho A$ is the beam's mass per unit length. $E$ is the Young modulus, $b$ and $h$ are the beam width and thickness, respectively. $c_{u}$ and $c_{w}$ are the viscous damping per unit length. $F(x, t)$ is the mechanical load, $I=b h^{3} / 12$ is the area moment of inertia and, $A=b h$ is the cross-section of the beam. $\Delta T$ is the temperature variation, and $\alpha$ the coefficient of thermal expansion. In the considered model, it is assumed that the temperature increases instantly, and the heat is uniformly distributed along the beam length and cross-section.

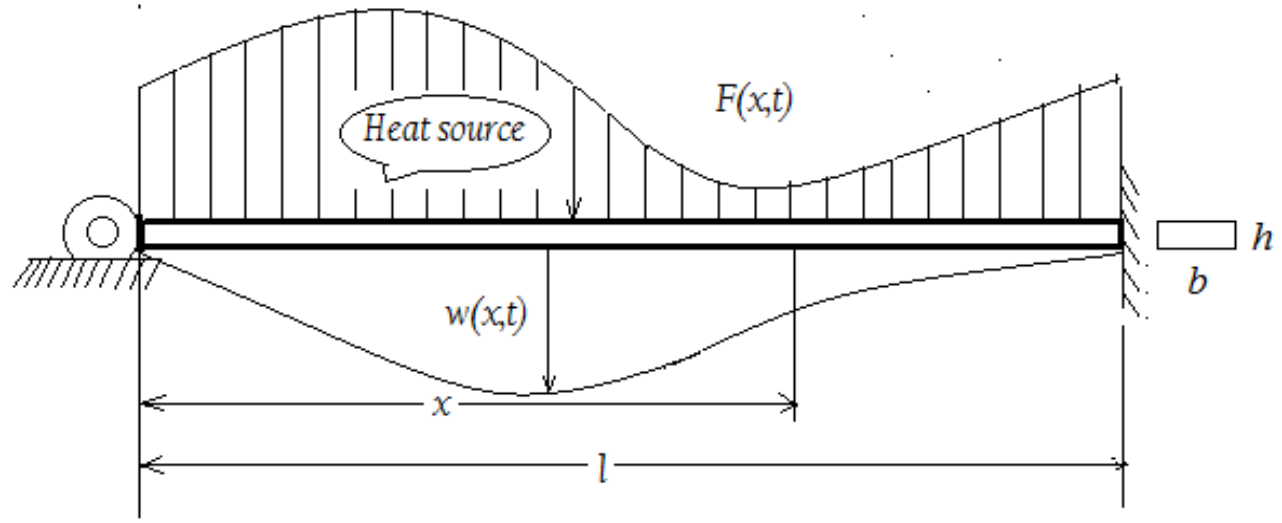

Fig. 1. Schematic diagram of a Hinged-Clamped beam submitted to simultaneous actions of thermal and mechanical loadings

The thermal stress-strain relation is given as follows:

$$
\gamma(\Delta T)=E A \alpha \Delta T
$$

The total strain in the beam is obtained by summing up the thermal and mechanical strains, that is:

$$
\varepsilon=\frac{\partial u}{\partial x}+\frac{1}{2}\left(\frac{\partial w}{\partial x}\right)^{2}-\frac{\gamma}{E}
$$

The extended Hamilton's principle reads:

$$
\int_{t_{1}}^{t_{2}}\left[\delta W_{e}-\delta T-\delta U\right] d t=0
$$

Where $W_{e}$ is the work done by external forces, $\mathrm{T}$ is the kinetic energy, and $\mathrm{U}$ is the elastic energy.

Hamilton's principle leads to the following PDEs of motion governing the thermoelastic behavior of the beam under-considered loadings.

$$
\begin{aligned}
m \frac{\partial^{2} u}{\partial t^{2}}+c_{u} \frac{\partial u}{\partial t}-A & \frac{\partial}{\partial x}\left\{E\left[\frac{\partial u}{\partial x}+\frac{1}{2}\left(\frac{\partial w}{\partial x}\right)^{2}\right]-\gamma\right\}=0 \\
m \frac{\partial^{2} w}{\partial t^{2}}+c_{w} \frac{\partial w}{\partial t}+ & E I \frac{\partial^{4} w}{\partial x^{4}} \\
& -A \frac{\partial}{\partial x}\left\{\frac{\partial w}{\partial x}\left\{E\left[\frac{\partial u}{\partial x}+\frac{1}{2}\left(\frac{\partial w}{\partial x}\right)^{2}\right]-\gamma\right\}\right\}=F(x) \cos (\Omega t)
\end{aligned}
$$


Where the mechanical load $F(x, t)$ has been assumed harmonic with spatial distribution $F(x)$ in the $w$ direction, and $\Omega$ is the excitation frequency.

Introducing the quasistatic assumptions, the acceleration and velocity terms in the $u$ direction are neglected [13]. This leads to:

$$
A \frac{\partial}{\partial x}\left\{E\left[\frac{\partial u}{\partial x}+\frac{1}{2}\left(\frac{\partial w}{\partial x}\right)^{2}\right]-\gamma\right\}=0
$$

One obtains the average strain of the system:

$$
\begin{aligned}
& \delta=\frac{1}{l} \int_{0}^{l}\left[\frac{\partial u}{\partial x}+\frac{1}{2}\left(\frac{\partial w}{\partial x}\right)^{2}-\frac{\gamma}{E}\right] d x \\
& =\frac{1}{2 l} \int_{0}^{l}\left(\frac{\partial w}{\partial x}\right)^{2} d x-\frac{\gamma}{E}
\end{aligned}
$$

Substituting (7) into (5), one obtains the following nonlinear partial differential equations of the planar motion.

$$
\begin{aligned}
E I \frac{\partial^{4} w}{\partial x^{4}}+m \frac{\partial^{2} w}{\partial t^{2}}= & -2 c \frac{\partial w}{\partial t}-A \gamma \frac{\partial^{2} w}{\partial x^{2}} \\
& +\frac{E A}{2 l} \frac{\partial^{2} w}{\partial x^{2}} \int_{0}^{l}\left(\frac{\partial w}{\partial x}\right)^{2} d x+F(x) \cos (\Omega t)
\end{aligned}
$$

The boundary conditions associated with equation (8) are:

$$
\begin{gathered}
w(0, t)=\frac{\partial^{2} w}{\partial x^{2}}(0, t)=0 \\
w(l, t)=\frac{\partial w}{\partial x}(l, t)=0
\end{gathered}
$$

Introducing the following non-dimensional quantities:

$$
\begin{gathered}
w^{*}=\frac{L}{r^{2}} w ; x^{*}=\frac{x}{L} ; t^{*}=\frac{t}{\tau} ; L=\frac{l}{2} ; l^{*}=\frac{l}{L}=2 ; r=\left(\frac{I}{A}\right)^{1 / 2} ; \varepsilon=\frac{r^{2}}{L^{2}} ; F^{*}=\frac{L^{7}}{r^{6} E A} F ; \\
\tau=\left(\frac{E r^{4}}{m L^{4}}\right)^{1 / 2} ; c^{*}=\frac{c}{2 A r^{3}}(m E)^{-1 / 2} ; v=1 / 4,
\end{gathered}
$$

and substituting (10) into (8) and (9) and omitting the asterisks, one obtains:

$$
\begin{gathered}
\frac{\partial^{4} w}{\partial x^{4}}+\frac{\partial^{2} w}{\partial t^{2}}=\varepsilon\left(-2 c \frac{\partial w}{\partial t}-\gamma^{T} \Delta T \frac{\partial^{2} w}{\partial x^{2}}+v \frac{\partial^{2} w}{\partial x^{2}} \int_{0}^{l}\left(\frac{\partial w}{\partial x}\right)^{2} d x+F \cos (\Omega t)\right) \\
w(0, t)=\frac{\partial^{2} w}{\partial x^{2}}(0, t) \\
w(l, t)=\frac{\partial w}{\partial x}(l, t)=0
\end{gathered}
$$

\section{Method}

Equations (11) can be solved approximately by Galerkin's method. The deflection is approximated by

$$
w(x, t)=\sum_{m=1}^{\infty} v_{m}(t) \varphi_{m}(x),
$$

Where $v_{m}$ are generalized coordinates and $\varphi_{m}$ are eigenfunctions of the following eigenvalue problem: 


$$
\begin{gathered}
\frac{d^{4} \varphi}{d x^{4}}-\omega_{m}^{2} \varphi_{m}=0 \quad \text { and } \\
\varphi_{m}(0)=0, \quad \frac{d^{2} \varphi_{m}}{d x^{2}}(0)=0, \\
\varphi_{m}(l)=0, \quad \frac{d \varphi_{m}}{d x}(l)=0,
\end{gathered}
$$

where $\omega_{m}$ are natural frequencies.

Substituting equation (12) into equations (11), multiplying by $\varphi_{n}$, integrating over the length and invoking orthogonality of the eigenfunctions, one obtains a set of ordinary nonlinear differential equations

$\frac{d^{2} u_{n}}{d t^{2}}+\omega_{n}{ }^{2} u_{n}=\varepsilon\left[-2 c_{n} \frac{d u_{n}}{d t}-\gamma_{n}{ }^{T} \Delta T u_{n}+F_{n} \cos (\Omega t)+\sum_{m, p, q=1}^{\infty} \alpha_{n m p q} u_{m} u_{p} u_{q}\right], n$

$=1,2, \ldots$

Where

$$
\begin{gathered}
c_{n}=\int_{0}^{l} c \varphi_{n}{ }^{2} d x, \\
\alpha_{n m p q}=v \int_{0}^{l} \varphi_{n}{ }^{2} \frac{d^{2} \varphi_{q}}{d x^{2}} d x \int_{0}^{l} \frac{d \varphi_{m}}{d x} \frac{d \varphi_{p}}{d x} d x, \\
F_{n}=\int_{0}^{l} F(x) \varphi_{n} v d x .
\end{gathered}
$$

The damping is assumed to be modal. The method of multiple scales can be used to construct a uniformly valid asymptotic expansion. According to this method, we assume that each $u_{n}$ is a function of different time scales which are defined by

$$
T^{n}=\varepsilon^{n} t
$$

And can be expanded in the form

$$
u_{n}(t ; \varepsilon)=\sum_{j=0}^{\infty} \varepsilon^{j} u_{n j}\left(T_{0}, T_{1}, \ldots\right), \quad n=1,2, \ldots
$$

Derivatives with respect to time transform according to

$$
\begin{aligned}
\frac{d}{d t} & =D_{0}+\varepsilon D_{1}+\cdots, \\
\frac{d^{2}}{d t^{2}} & =D_{0}^{2}+\varepsilon^{2} D_{0} D_{1}+\cdots,
\end{aligned}
$$

Where

$$
D_{0}=\frac{\partial}{\partial T_{0}}, \quad D_{1}=\frac{\partial}{\partial T_{1}}, \ldots
$$

Substituting equations (15) into equations (14) and equating coefficients of like powers of $\varepsilon$, we obtain

$$
\varepsilon^{0}: D_{0}{ }^{2} u_{n 0}+\omega_{n}{ }^{2} u_{n 0}=0, \quad n=1,2, \ldots
$$




$$
\begin{aligned}
\varepsilon^{1}: D_{1}{ }^{2} u_{n 1}+\omega_{n}{ }^{2} u_{n 1}= & -2 D_{0} D_{1} u_{n 0}-2 c_{n} D_{0} u_{n 0}-\gamma_{n}{ }^{T} \Delta T u_{n 0} \\
& +F_{n} \cos \left(\Omega T_{0}\right)+\sum_{m, p, q=1}^{\infty} \alpha_{n m p q} u_{m 0} u_{p 0} u_{q 0}, \quad n=1,2, \ldots
\end{aligned}
$$

The solution of equation (16) can be written as follows

$$
u_{n 0}=A_{n}\left(T_{1}, T_{2}, \ldots\right) \exp \left(i \omega_{n} T_{0}\right)+c c, \quad n=1,2, \ldots
$$

where $c c$ stands for the complex conjugate of the terms to the left. At this point, the $A_{n}$ are unknown. These are determined from the elimination of secular terms at the next level of the approximation. Substituting equations (18) into (17) leads to

$$
\begin{aligned}
D_{1}{ }^{2} u_{n 1}+\omega_{n}{ }^{2} u_{n 1} & -2 i \omega_{n}\left(D_{1} A_{n}+c_{n} A_{n}\right) \exp \left(i \omega_{n} T_{0}\right)-\gamma_{n}{ }^{T} \Delta T A_{n} \exp \left(i \omega_{n} T_{0}\right) \\
& +\frac{1}{2} F_{n} \exp \left(i \Omega T_{0}\right) \\
& +\sum_{m, p, q=1}^{\infty} \alpha_{n m p q}\left\{A_{m} A_{p} A_{q} \exp \left[i\left(\omega_{m}+\omega_{P}+\omega_{q}\right) T_{0}\right]\right. \\
& +A_{m} A_{p} \bar{A}_{q} \exp \left[i\left(\omega_{m}+\omega_{P}-\omega_{q}\right) T_{0}\right]+A_{m} \bar{A}_{p} A_{q} \exp \left[i\left(\omega_{m}-\omega_{P}+\omega_{q}\right) T_{0}\right] \\
& \left.+A_{m} \bar{A}_{p} \bar{A}_{q} \exp \left[i\left(\omega_{m}-\omega_{P}-\omega_{q}\right) T_{0}\right]\right\}+c c, n \\
& =1,2, \ldots .
\end{aligned}
$$

In order to eliminate the secular terms from $u_{n 1}$, the $A_{n}$ must be chosen so that the coefficient of $\exp \left(i \omega_{n} T_{0}\right)$ is zero. This coefficient will contain $F_{n}$ when $\Omega$ is near $\omega_{n}$ as well as the nonlinear terms associated with any combinations of the form

$$
\omega_{n} \approx \pm \omega_{m} \pm \omega_{P} \pm \omega_{q}
$$

The eigenfunction of the eigenvalue problem (13) is

$$
\varphi_{n}=E_{n}\left[\sin \left(\alpha_{n} x\right)-R_{n} \sinh \left(\alpha_{n} x\right)\right]
$$

Where

$$
\begin{gathered}
E_{n}=\left[\frac{1}{2} l\left(1-R_{n}{ }^{2} \sinh \left(2 \alpha_{n} l\right)-\sin \left(2 \alpha_{n} l\right)\right) / 4 \alpha_{n}\right]^{-1 / 2} \\
R_{n}=\sin \left(\alpha_{n} l\right) / \sinh \left(\alpha_{n} l\right), \quad \alpha_{n} l=\omega_{n}{ }^{1 / 2}
\end{gathered}
$$

And $\alpha_{n}$ are the roots of $\tan \left(\alpha_{n} l\right)=\tanh \left(\alpha_{n} l\right)$.

In the case of $l=2$, the first three roots and frequencies are

$$
\begin{gathered}
\alpha_{1} l=3.927 \quad \text { and } \omega_{1}=15.421 \\
\alpha_{2} l=7.069 \quad \text { and } \omega_{2}=49.970 \\
\alpha_{3} l=10.210 \quad \text { and } \quad \omega_{1}=104.24
\end{gathered}
$$

From the observation of natural frequencies, we can see the relations $\omega_{2} \approx 3 \omega_{1}$ and $\omega_{3} \approx$ $\omega_{1}+2 \omega_{2}$, which satisfies the condition of internal resonance condition (20). We introduce detuning parameters $\sigma_{12}$ and $\sigma_{13}$ as follows:

$$
\begin{aligned}
& \omega_{2}=3 \omega_{1}\left(1+\varepsilon \sigma_{12}\right), \\
& \omega_{3}=\left(\omega_{1}+2 \omega_{2}\right)\left(1+\varepsilon \sigma_{13}\right),
\end{aligned}
$$

where 


$$
\varepsilon \sigma_{12}=0.0801, \varepsilon \sigma_{13}=-0.0964 .
$$

In this study we consider primary resonances. To express the nearness of $\Omega$ to $\omega_{s}$, we introduce a detuning parameter $\sigma_{2}$ as follows:

$$
\Omega=\omega_{s}\left(1+\varepsilon \sigma_{2}\right), \text { for } s=1,2, \ldots
$$

Substituting the resonance conditions (21) and (22) into equations (19), and eliminating the secular terms, we have the following solvability conditions

$$
\begin{aligned}
& -2 i \omega_{1}\left(A_{1}^{\prime}+c_{1} A_{1}\right)-\gamma_{1}{ }^{T} \Delta T A_{1}+A_{1} \sum_{j=1}^{\infty} \gamma_{1 j} A_{j} \bar{A}_{j}+q_{3} \bar{A}_{1}{ }^{2} A_{2} \exp \left(i 3 \omega_{1} \sigma_{12} T_{1}\right) \\
& +q_{7}{\overline{A_{2}}}^{2} A_{3} \exp \left(i\left(\omega_{1}+2 \omega_{2}\right) \sigma_{13} T_{1}\right) \\
& +\frac{1}{2} F_{1} \delta_{1 s} \exp \left(i \sigma_{2} \omega_{1} T_{1}\right)=0 \\
& -2 i \omega_{2}\left(A_{2}^{\prime}+c_{2} A_{2}\right)-{\gamma_{2}}^{T} \Delta T A_{2}+A_{2} \sum_{j=1}^{\infty} \gamma_{2 j} A_{j} \bar{A}_{j}+q_{6} A_{1}{ }^{3} \exp \left(-i 3 \omega_{1} \sigma_{12} T_{1}\right) \\
& +q_{8} \bar{A}_{1} \bar{A}_{2} A_{3} \exp \left(i\left(\omega_{1}+2 \omega_{2}\right) \sigma_{13} T_{1}\right) \\
& +\frac{1}{2} F_{2} \delta_{2 s} \exp \left(i \sigma_{2} \omega_{2} T_{1}\right)=0 \\
& -2 i \omega_{3}\left(A_{3}^{\prime}+c_{3} A_{3}\right)-\gamma_{3}{ }^{T} \Delta T A_{3}+A_{3} \sum_{j=1}^{\infty} \gamma_{3 j} A_{j} \bar{A}_{j}+q_{9} A_{1} A_{2}{ }^{2} \exp \left(-i\left(\omega_{1}+2 \omega_{2}\right) \sigma_{13} T_{1}\right) \\
& +\frac{1}{2} F_{3} \delta_{3 s} \exp \left(i \sigma_{2} \omega_{3} T_{1}\right)=0 \\
& -2 i \omega_{n}\left(A_{n}^{\prime}+c_{n} A_{n}\right)-\gamma_{n}{ }^{T} \Delta T A_{n}+A_{n} \sum_{j=1}^{\infty} \gamma_{n j} A_{j} \bar{A}_{j} \\
& +\frac{1}{2} F_{n} \delta_{n s} \exp \left(i \sigma_{2} \omega_{n} T_{1}\right)=0, n \geq 4
\end{aligned}
$$

The coefficients $\gamma_{n j}$ and $q_{k}$ can be found in [6], and $\delta_{3 s}$ is the Kronecker delta.

To solve equations (23), we write $A_{n}$ in the polar form

$$
A_{n}=\frac{1}{2} a_{n} \exp \left(i \omega_{1} \beta_{n}\right), \quad n=1,2, \ldots
$$

Where $a_{n}$ and $\beta_{n}$ are real. Then we separate the result into its real and imaginary parts and obtain

$n=1,2,3:$

$$
\begin{gathered}
\omega_{1}\left(a_{1}^{\prime}+c_{1} a_{1}\right)-\frac{1}{8}\left(q_{3} a_{1}^{2} a_{2} \sin \mu_{1}+q_{7} a_{2}{ }^{2} a_{3} \sin \mu_{3}\right)-\frac{1}{2} F_{1} \delta_{1 s} \sin \mu_{2}=0 \\
\omega_{1}{ }^{2} a_{1} \beta_{1}^{\prime}+\frac{1}{8}\left(q_{1} a_{1}{ }^{3}+q_{2} a_{1} a_{2}{ }^{2}+q_{3} a_{1}{ }^{2} a_{2} \cos \mu_{1}+q_{7} a_{2}{ }^{2} a_{3} \cos \mu_{3}+q_{10} a_{1} a_{3}{ }^{2}\right. \\
\left.+a_{1} \sum_{j=4}^{\infty} \gamma_{1 j} a_{j}{ }^{2}\right)-\frac{1}{2} \gamma_{1}{ }^{T} \Delta T a_{1}+\frac{1}{2} F_{1} \delta_{1 s} \cos \mu_{2} \\
=0
\end{gathered}
$$


$\omega_{2}\left(a_{2}^{\prime}+c_{2} a_{2}\right)+\frac{1}{8}\left(q_{6} a_{1}{ }^{3} \sin \mu_{1}-q_{8} a_{1} a_{2} a_{3} \sin \mu_{3}\right)-\frac{1}{2} F_{2} \delta_{2 s} \sin \mu_{2}=0$

$\omega_{1} \omega_{2} a_{2} \beta_{2}^{\prime}$

$+\frac{1}{8}\left(q_{4} a_{2}{ }^{3}+q_{5} a_{1}{ }^{2} a_{2}+q_{6} a_{1}{ }^{3} \cos \mu_{1}+q_{8} a_{1} a_{2} a_{3} \cos \mu_{3}+q_{11} a_{2} a_{3}{ }^{2}+a_{2} \sum_{j=4}^{\infty} \gamma_{2 j} a_{j}{ }^{2}\right)$

$-\frac{1}{2} \gamma_{2}^{T} \Delta T a_{2}+\frac{1}{2} F_{2} \delta_{2 s} \cos \mu_{2}$

$=0$

$\omega_{3}\left(a_{3}^{\prime}+c_{3} a_{3}\right)+\frac{1}{8} q_{9} a_{1} a_{2}{ }^{2} \sin \mu_{3}-\frac{1}{2} F_{3} \delta_{3 s} \sin \mu_{2}=0$

$\omega_{1} \omega_{3} a_{3} \beta_{3}^{\prime}+\frac{1}{8}\left(q_{9} a_{1} a_{2}{ }^{2} \cos \mu_{3}+q_{12} a_{1}{ }^{2} a_{3}+q_{13} a_{2}{ }^{2} a_{3}+q_{14} a_{3}{ }^{3}+a_{3} \sum_{j=4}^{\infty} \gamma_{3 j} a_{j}{ }^{2}\right)-\frac{1}{2} \gamma_{3}{ }^{T} \Delta T a_{3}$

$+\frac{1}{2} F_{3} \delta_{3 s} \cos \mu_{2}$

$=0$

$n \geq 4:$

$a_{n}^{\prime}+c_{n} a_{n}-\frac{1}{2} F_{n} \delta_{n s} \sin \mu_{2}=0$

$\omega_{1} \omega_{n} a_{n} \beta_{n}^{\prime}-\frac{1}{2} \gamma_{n}^{T} \Delta T a_{n}+\frac{1}{8} a_{n} \sum_{j=1}^{\infty} \gamma_{n j} a_{j}^{2}+\frac{1}{2} F_{n} \delta_{n s} \cos \mu_{2}=0$,

Where

$$
\begin{gathered}
\mu_{1}=\omega_{1}\left(3 \sigma_{12} T_{1}-3 \beta_{1}+\beta_{2}\right), \\
\mu_{2}=\omega_{s} \sigma_{2} T_{1}-\omega_{1} \beta_{s}, \\
\mu_{3}=\left(\omega_{1}+2 \omega_{2}\right) \sigma_{13} T_{1}+\omega_{1} \beta_{3}-2 \omega_{1} \beta_{2}-\omega_{1} \beta_{1},
\end{gathered}
$$

We consider the case of interest $s=1,2,3$. These cases are discussed separately.

Case I: $\Omega \approx \omega_{1} \quad(s=1)$

It follows from equation $(25 \mathrm{~g})$ that

$$
a_{n}^{\prime}+c_{n} a_{n}=0, \quad n \geq 4 .
$$

The amplitudes $a_{n}$ decay because

$$
a_{n} \propto \exp \left(-c_{n} T_{1}\right), \quad n \geq 4 .
$$

Since we are interested in the steady-state response, we can disregard $a_{n}(n \geq 4)$. then equations (25a)-(25f) can be reduced to a set of autonomous ordinary differential equations in amplitudes $a_{n}$ and phase $\mu_{n}$ for $n=1,2,3$. letting $a_{1}^{\prime}=a_{2}^{\prime}=a_{3}^{\prime}=\mu_{1}^{\prime}=\mu_{2}^{\prime}=\mu_{3}^{\prime}=0$, one obtains the following algebraic equations giving the steady-state responses.

$\omega_{1} c_{1} a_{1}-\frac{1}{8}\left(q_{3} a_{1}^{2} a_{2} \sin \mu_{1}+q_{7} a_{2}^{2} a_{3} \sin \mu_{3}\right)-\frac{1}{2} F_{1} \sin \mu_{2}=0$ 


$$
\begin{gathered}
\omega_{1}^{2} \sigma_{2} a_{1}-\frac{1}{2} \gamma_{1}{ }^{T} \Delta T a_{1}+\frac{1}{8}\left(q_{1} a_{1}^{3}+q_{2} a_{1} a_{2}{ }^{2}+q_{3} a_{1}{ }^{2} a_{2} \cos \mu_{1}+q_{7} a_{2}{ }^{2} a_{3} \cos \mu_{3}+q_{10} a_{1} a_{3}{ }^{2}\right) \\
+\frac{1}{2} F_{1} \cos \mu_{2}=0
\end{gathered}
$$

$$
\omega_{2} c_{2} a_{2}+\frac{1}{8}\left(q_{6} a_{1}{ }^{3} \sin \mu_{1}-q_{8} a_{1} a_{2} a_{3} \sin \mu_{3}\right)=0
$$

$3 \omega_{1} \omega_{2} a_{2}\left(\sigma_{2}-\sigma_{12}\right)-\frac{1}{2} \gamma_{2}{ }^{T} \Delta T a_{2}$

$$
+\frac{1}{8}\left(q_{4} a_{2}{ }^{3}+q_{5} a_{1}{ }^{2} a_{2}+q_{6} a_{1}{ }^{3} \cos \mu_{1}+q_{8} a_{1} a_{2} a_{3} \cos \mu_{3}+q_{11} a_{3}{ }^{2} a_{2}\right)=0
$$

$$
\omega_{3} c_{3} a_{3}+\frac{1}{8} q_{9} a_{1} a_{2}{ }^{2} \sin \mu_{3}=0
$$

$$
\begin{aligned}
& \omega_{1} \omega_{3} a_{3}\left[7 \sigma_{2}-6 \sigma_{12}-\frac{\left(\omega_{1}+2 \omega_{2}\right) \sigma_{13}}{\omega_{1}}\right]-\frac{1}{2} \gamma_{3}{ }^{T} \Delta T a_{3} \\
& +\frac{1}{8}\left(q_{9} a_{1} a_{2}{ }^{2} \cos \mu_{3}+q_{12} a_{1}^{2} a_{3}+q_{13} a_{2}{ }^{2} a_{3}+q_{14} a_{3}{ }^{3}\right)=0
\end{aligned}
$$

Case II: $\Omega \approx \omega_{2}(s=2)$.

Proceeding in a similar way, we obtain the following algebraic equations giving the steadystate responses.

$$
\begin{aligned}
& \omega_{1} c_{1} a_{1}-\frac{1}{8}\left(q_{3} a_{1}^{2} a_{2} \sin \mu_{1}+q_{7} a_{2}^{2} a_{3} \sin \mu_{3}\right)=0 \\
& \omega_{1} a_{1}\left(\omega_{2} \sigma_{2}+3 \omega_{1} \sigma_{12}\right)-\frac{1}{2} \gamma_{1}^{T} \Delta T a_{1} \\
& +\frac{3}{8}\left(q_{1} a_{1}^{3}+q_{2} a_{1} a_{2}{ }^{2}+q_{3} a_{1}{ }^{2} a_{2} \cos \mu_{1}+q_{7} a_{2}{ }^{2} a_{3} \cos \mu_{3}+q_{10} a_{1} a_{3}{ }^{2}\right) \\
& =0 \\
& \omega_{2} c_{2} a_{2}+\frac{1}{8}\left(q_{6} a_{1}{ }^{3} \sin \mu_{1}-q_{8} a_{1} a_{2} a_{3} \sin \mu_{3}\right)-\frac{1}{2} F_{2} \sin \mu_{2}=0 \\
& \omega_{2}^{2} a_{2} \sigma_{2}-\frac{1}{2} \gamma_{2}{ }^{T} \Delta T a_{2} \\
& \begin{array}{l}
+\frac{1}{8}\left(q_{4} a_{2}{ }^{3}+q_{5} a_{1}{ }^{2} a_{2}+q_{6} a_{1}{ }^{3} \cos \mu_{1}+q_{8} a_{1} a_{2} a_{3} \cos \mu_{3}+q_{11} a_{2} a_{3}{ }^{2}\right) \\
+\frac{1}{2} F_{2} \cos \mu_{2}=0
\end{array} \\
& \omega_{3} c_{3} a_{3}+\frac{1}{8} q_{9} a_{1} a_{2}^{2} \sin \mu_{3}=0 \\
& \omega_{3} a_{3}\left[\left(7 \omega_{2} \sigma_{2}+3 \omega_{1} \sigma_{12}\right)-3\left(\omega_{1}+2 \omega_{2}\right) \sigma_{13}\right]-\frac{1}{2} \gamma_{3}{ }^{T} \Delta T a_{3} \\
& +\frac{3}{8}\left(q_{9} a_{1} a_{2}{ }^{2} \cos \mu_{3}+q_{12} a_{1}{ }^{2} a_{3}+q_{13} a_{2}{ }^{2} a_{3}+q_{14} a_{3}{ }^{3}=0\right)
\end{aligned}
$$


Case III: $\Omega \approx \omega_{3} \quad(s=3)$.

The following algebraic equations are obtained when the excitation frequency is near the natural frequency of the third mode

$$
\begin{gathered}
\omega_{1} c_{1} a_{1}-\frac{1}{8}\left(q_{3} a_{1}{ }^{2} a_{2} \sin \mu_{1}+q_{7} a_{2}{ }^{2} a_{3} \sin \mu_{3}\right)=0 \\
\omega_{1} a_{1}\left[\left(\omega_{1}+2 \omega_{2}\right) \sigma_{13}+6 \omega_{1} \sigma_{12}+\omega_{3} \sigma_{2}\right]-\frac{1}{2} \gamma_{1}{ }^{T} \Delta T a_{1} \\
+\frac{7}{8}\left(q_{1} a_{1}{ }^{3}+q_{2} a_{1} a_{2}{ }^{2}+q_{3} a_{1}{ }^{2} a_{2} \cos \mu_{1}+q_{7} a_{2}{ }^{2} a_{3} \cos \mu_{3}+q_{10} a_{1} a_{3}{ }^{2}\right) \\
=0 \quad(29 b) \\
3 \omega_{2} a_{2}\left[\sigma_{13}\left(\omega_{1}+2 \omega_{2}\right)+\omega_{3} \sigma_{2}-\omega_{1} \sigma_{12}\right]-\frac{1}{2} \gamma_{2}{ }^{T} \Delta T a_{2} \\
+\frac{7}{8}\left(q_{4} a_{2}{ }^{3}+q_{5} a_{1}{ }^{2} a_{2}+q_{6} a_{1}{ }^{3} \cos _{1}+q_{8} a_{1} a_{2} a_{3} \cos \mu_{3}+q_{11} a_{2} a_{3}{ }^{2}\right) \\
=0
\end{gathered}
$$

Equations (27)-(29) are systems of nonlinear algebraic equations with six unknowns, $a_{1}$, $a_{2}, a_{3}, \mu_{1}, \mu_{2}, \mu_{3}$ and one parameter $\sigma_{2} . a_{i}(i=1,2,3)$ are steady states amplitudes of the three involved modes while $\mu_{i}(i=1,2,3)$ represent the corresponding phases. These equation systems are solved by the mean of the Newton-Raphson shooting technique for which the flowchart is presented in Fig. 2.

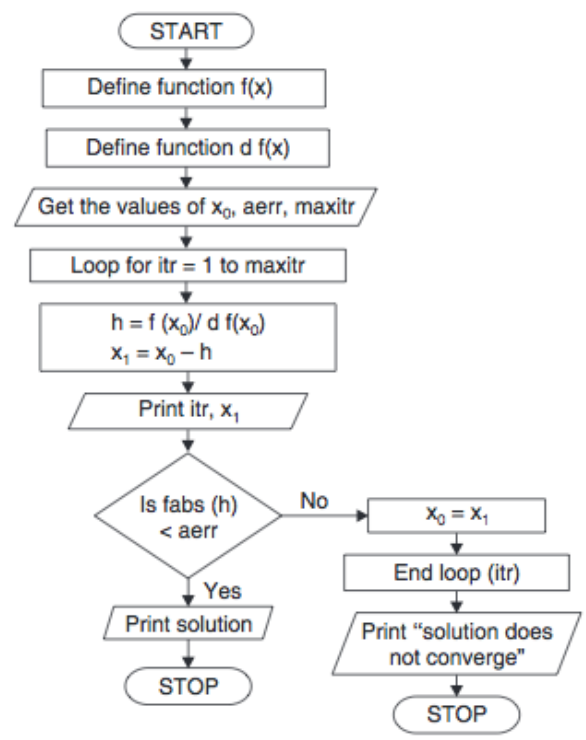

Fig. 2. Flowchart of Newton-Raphson shooting technique 


\section{Results and Discussion}

\subsection{Temperature-free responses}

At room temperature, the resolution of systems of equations (27)-(29) leads to the following results in agreement with the work done by authors of papers [5]-[7]. The frequency response curves are presented in figures (2)-(4) for excitation frequency near the frequency of the first mode and in figures (5)-(6) for excitation frequency near the frequency of the second mode. When the excitation frequency is near the frequency of the first mode, the response amplitude is dominated by the first mode (Fig. 3) with a small amplitude for the second mode (Fig. 4) and a very weak amplitude for the third mode (Fig. 5). When the second mode is excited, Fig. 6 shows that there is a range of excitation frequency for which the response is dominated by the first mode (internal resonance). The third mode still presents small amplitude (Fig. 7).

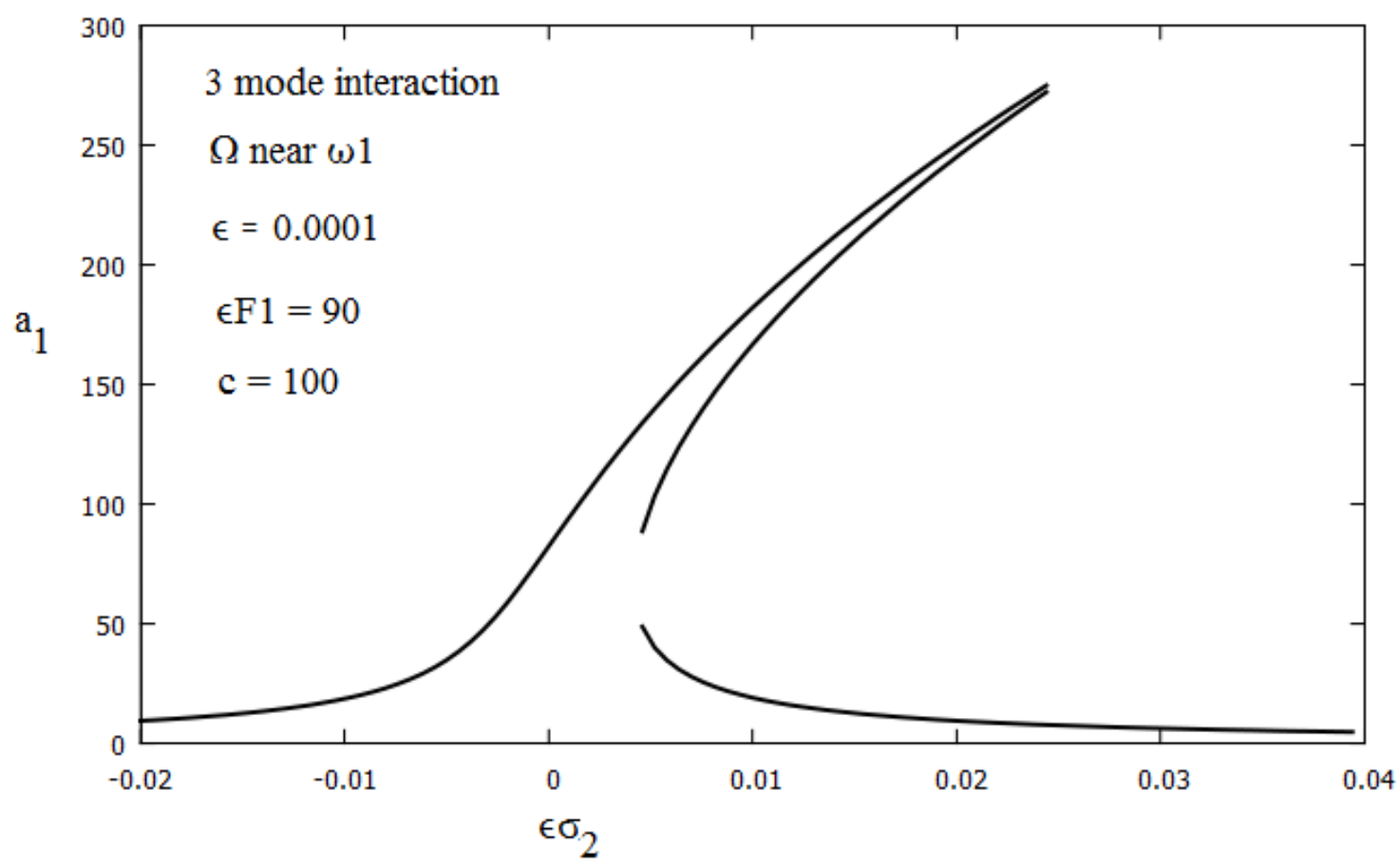

Fig. 3. Variation of amplitude a 1 of the first mode with excitation frequency when the first mode is excited $\left(\Omega \approx \omega_{1}\right)$ at room temperature, $\Delta \mathrm{T}=0$.

\subsection{Responses under temperature change}

Non-free thermal stress responses are presented in this section. In the presence of temperature change, nonlinear algebraic systems of equations (27)-(29) are solved by the mean of Newton-Raphson shooting technique to investigate the thermal effects on the response amplitude of each of the three modes involved when one of the modes is directly excited. All figures presenting temperature-influenced responses are depicted alongside the corresponding temperature-free response in order to appreciate the temperature effect on these responses.

When the excitation frequency is near the frequency of the first mode, Fig. $8 \mathrm{a}$ and Fig. 8b present the effect of temperature on the amplitude response of the first mode respectively for $\Delta T= \pm 30^{\circ} \mathrm{C}$ and $\Delta T= \pm 100^{\circ} \mathrm{C}$. It is globally observed a shift in frequency along the frequency axis, to the left for positive temperature difference and to the right for negative one 
in comparison to the temperature-free curve $\left(\Delta T=0{ }^{\circ} \mathrm{C}\right)$. These results agree with those obtained by authors of papers [10]-[13]. The shift increases with the absolute value of temperature difference. There is no notable change, however, in amplitude between temperature-free response and responses under the thermal influence in this case.

Fig. 9a and Fig. 9b present the effect of temperature change on the response amplitude of the second mode for temperature difference $\Delta T= \pm 50{ }^{\circ} \mathrm{C}$ and $\Delta T= \pm 100^{\circ} \mathrm{C}$ respectively. Similar observation as previously made on the responses of the first mode in terms of a shift in frequency domain due to temperature change can be made here. However, there is a change in regard to the maximal amplitude reached for different responses. In comparison with the temperature-free response curve, amplitudes are larger for negative temperature difference and lower for positive one, for the discussed case of the second mode responding when the first mode is directly excited. As a quantitative example, figure 8a presents maximal amplitude of about $2.5,1.5$, and 0.8 respectively for $\Delta T=-50{ }^{\circ} \mathrm{C}, \Delta T=0{ }^{\circ} \mathrm{C}$, and $\Delta T=+50^{\circ} \mathrm{C}$. These values turn into around $4.5,1.5$, and 0.6 respectively for $\Delta T=-100^{\circ} \mathrm{C}, \Delta T=0{ }^{\circ} \mathrm{C}$, and $\Delta T=$ $+100^{\circ} \mathrm{C}$ in Fig. 9b. When the excitation frequency is near the frequency of the second mode, Fig. 10a - Fig. 10c show the effect of temperature change on the response amplitude of the first mode, Fig. 11a - Fig. 11c present the same results for the second mode, while Fig. 12a and Fig. $12 \mathrm{~b}$ depict these results for the third mode.

For the first mode (Fig. 10a - Fig. 10c), results show the previously described shift in the frequency domain of the response amplitude for negative and positive temperature change on either side of the thermal free-response curve. Nonetheless, there is a change in amplitude for different curves. However, in opposition to the aforementioned case, higher amplitudes are observed for positive temperature difference and smaller for negative one as compared with room temperature response. Qualitative change is also observed between these response amplitude curves, and it can be seen that as positive temperature difference increases, the profile of the response curve gradually loses its resonant profile.

The responses of the second mode of vibration when the same mode is excited in the presence of thermal influence are presented in figures 10a-10c. One notes the shift in frequency caused by the temperature difference on either side of the room temperature response depending on the sign of temperature difference. In addition, from a quantitative point of view, it is observed that the temperature free-response curve presents higher amplitude over the temperature influenced response curves. This situation is quite different from those observed previously. The responses of the third mode (Fig. 12a and Fig. 12b) reacting to the second mode directly excited in the presence of temperature change globally present the same behavior as described for the response of the second mode, but with weak amplitude.

These results clearly state that temperature influence on the responses of a hingedclamped beam undergoing nonlinear vibration under a periodic mechanical excitation is important. This influence is diversely observed on the different substructures when multimodal interaction is considered. The changes are observed both qualitatively and quantitatively and are not the same on the different substructures when one of the modes is directly excited. These outcomes show that one should pay more attention when designing structural elements involving vibrational behavior under simultaneous actions of mechanical excitation and thermal loads. So far, temperature-free analysis has revealed that when multimodal interaction is involved in a hinged-clamped beam vibration, there is a possibility of internal resonance with modes other than the directly excited mode responding with higher amplitude in some frequency interval due to energy transfer in between the interacting substructures.

Actually, it is shown that the effect of temperature mistakenly evaluated as a flat rate in several structural and civil engineering designs needs to be paid more attention as far as multimodal interaction is involved under the action of mechanical excitation. There is a shift in 
the frequency domain of the thermal influenced responses on either side of the temperaturefree response curve, depending on the magnitude and sign of the temperature difference. Moreover, when a given mode is directly excited, higher amplitudes are obtained as function of the responding substructure, the sign, and the magnitude of the temperature difference. It has been seen that for some substructures, the larger amplitude is obtained for negative temperature difference, while it is observed for positive temperature difference for others and for temperature-free responses for some others. This inspires the existence of a very rich dynamic of the studied system to be investigated in future work.

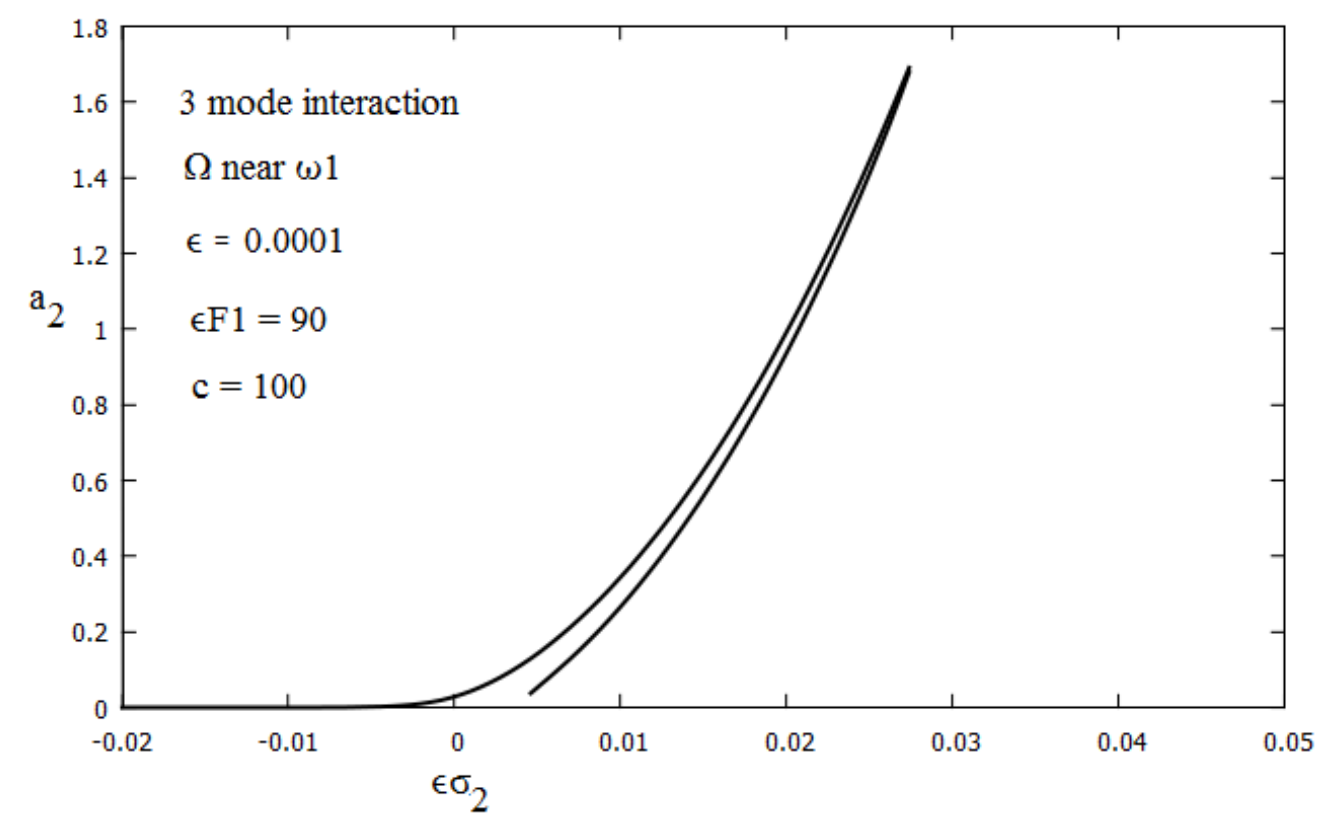

Fig. 4. Variation of amplitude a 2 of the second mode with excitation frequency when the first mode is excited $\left(\Omega \approx \omega_{1}\right)$ at room temperature, $\Delta \mathrm{T}=0$

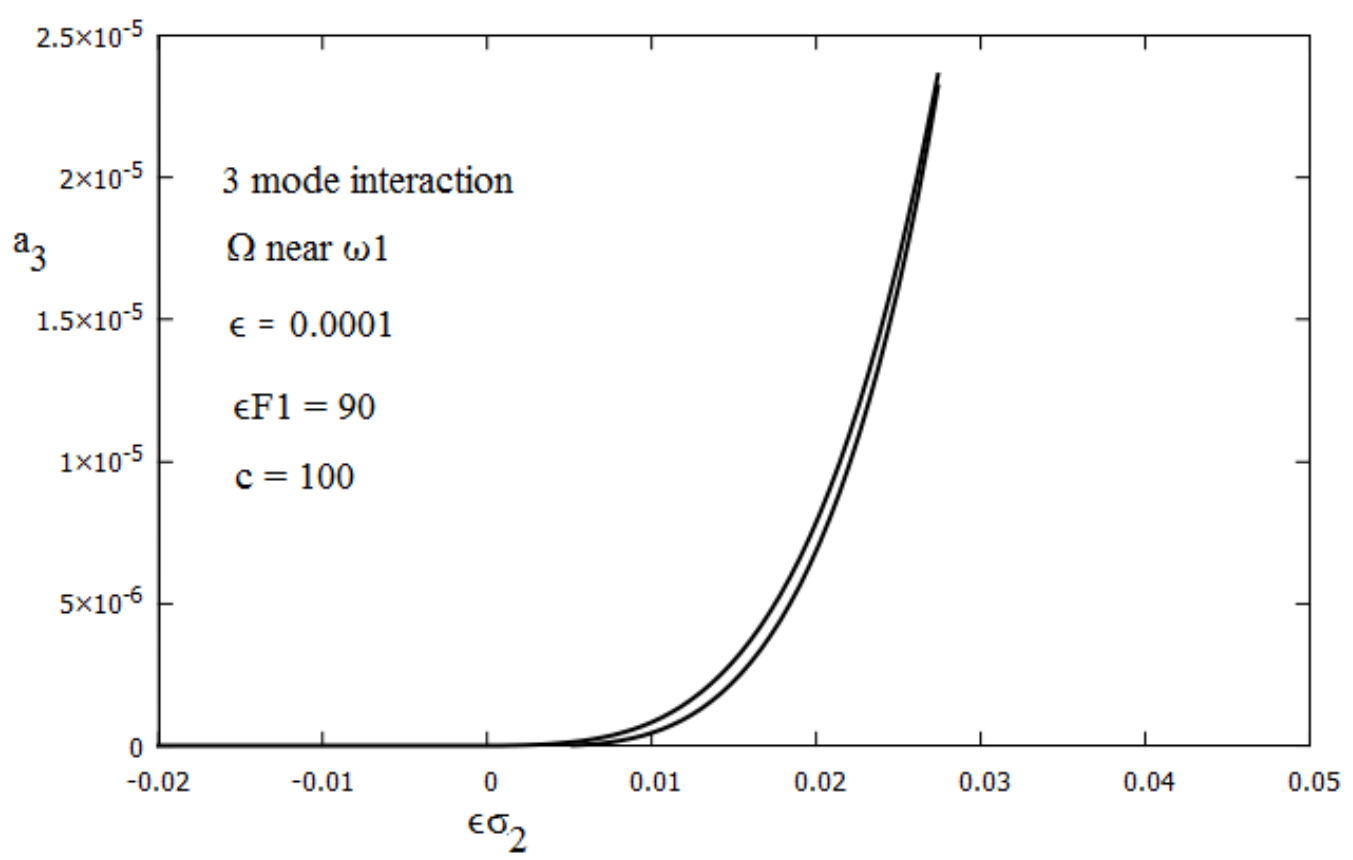

Fig. 5. Variation of amplitude a 3 of the third mode with excitation frequency when the first mode is excited $\left(\Omega \approx \omega_{1}\right)$ at room temperature, $\Delta \mathrm{T}=0$ 


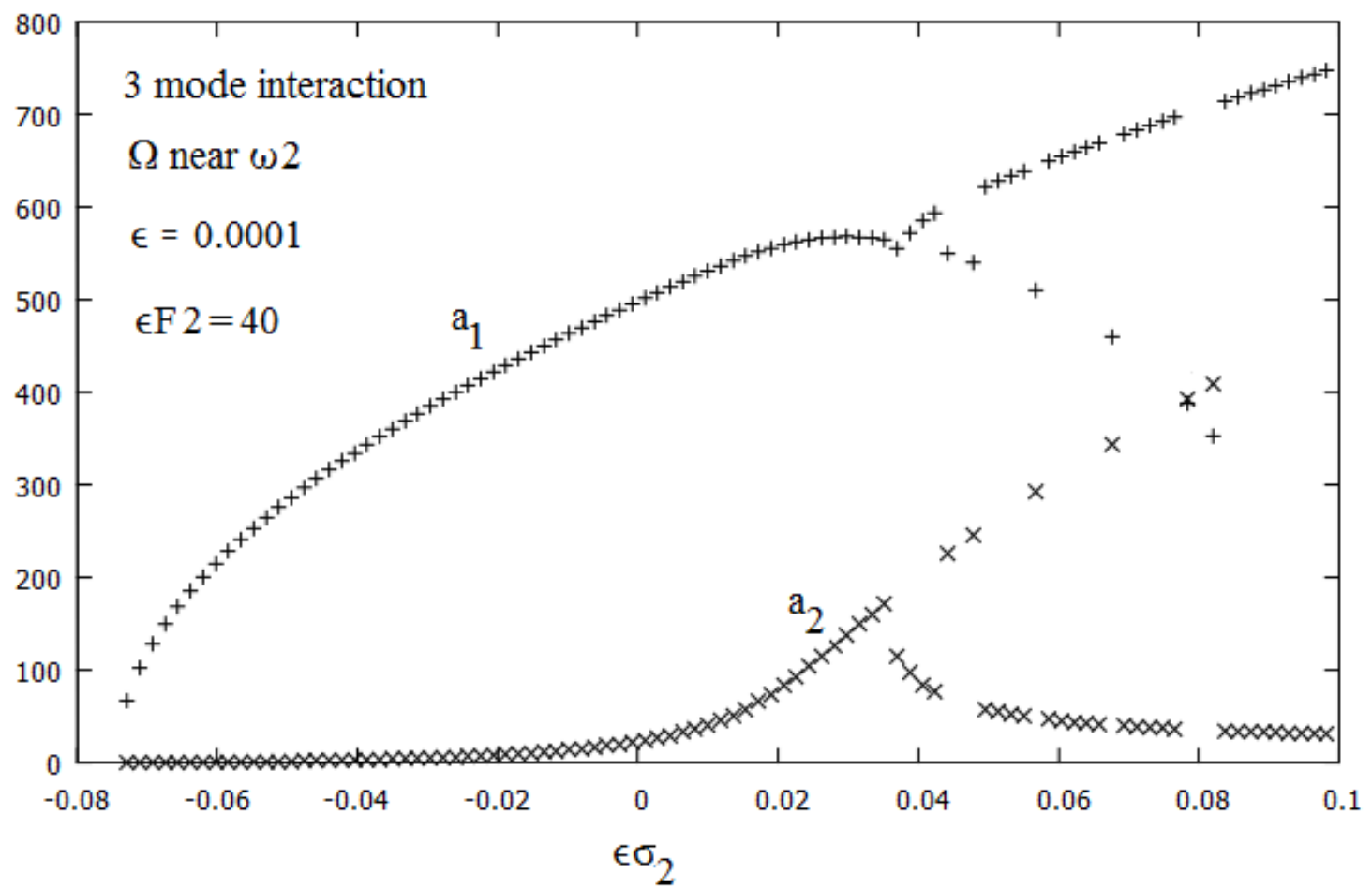

Fig. 6. Variation of amplitudes $\mathrm{a} 1$ and $\mathrm{a} 2$ of first and second mode with excitation frequency when the second mode is excited $\left(\Omega \approx \omega_{2}\right)$ at room temperature, $\Delta \mathrm{T}=0$

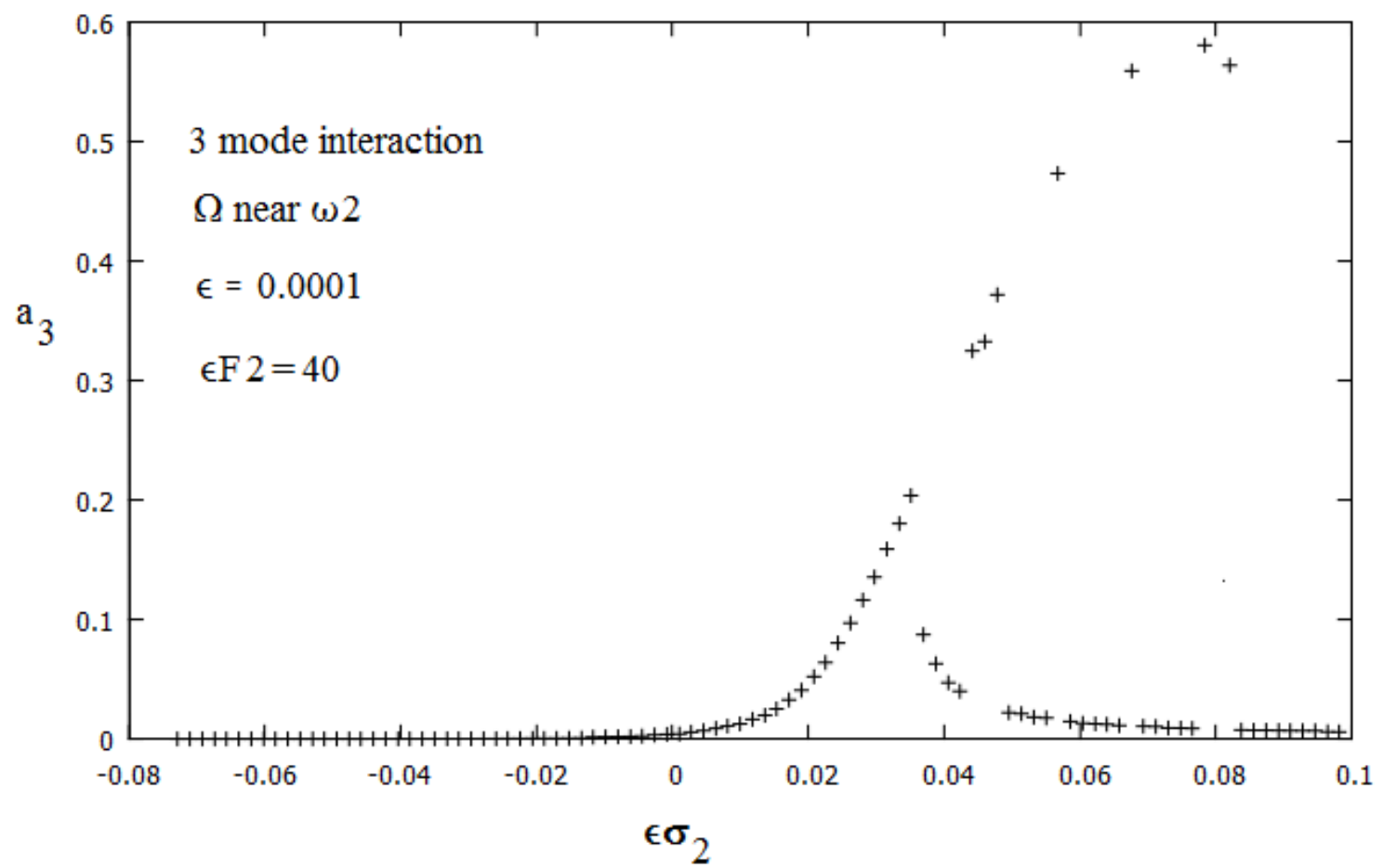

Fig. 7. Variation of amplitude a3 of third mode with excitation frequency when the second mode is excited $\left(\Omega \approx \omega_{2}\right)$ at room temperature, $\Delta \mathrm{T}=0$ 


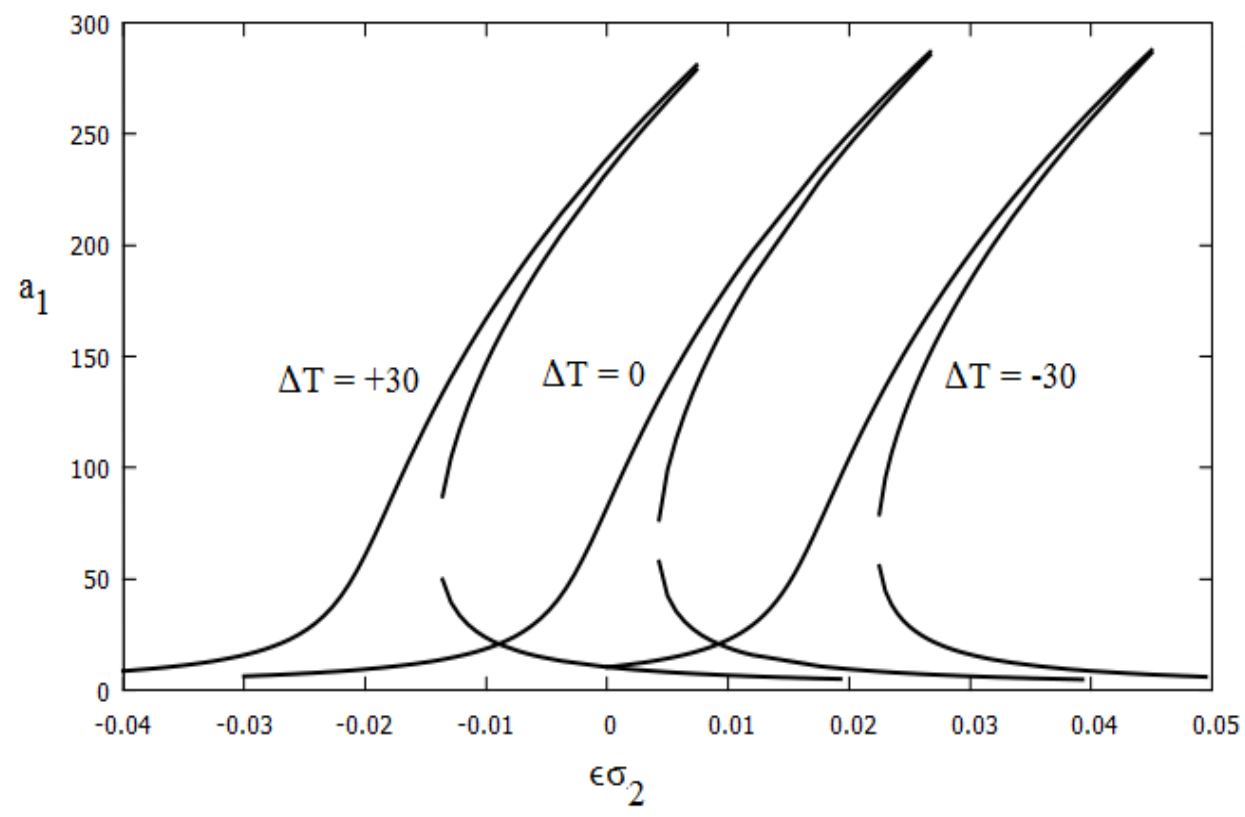

3 mode interaction

$\Omega$ near $\omega 1$

$\epsilon=0.0001$

$\epsilon \mathrm{F} 1=90$

$\mathrm{c}=100$

Fig. 8a. Variation of amplitude a1 of first mode with excitation frequency when the first mode is excited $\left(\Omega \approx \omega_{1}\right)$ at temperatures $\Delta \mathrm{T}=+30, \Delta \mathrm{T}=0$ and $\Delta \mathrm{T}=-30$

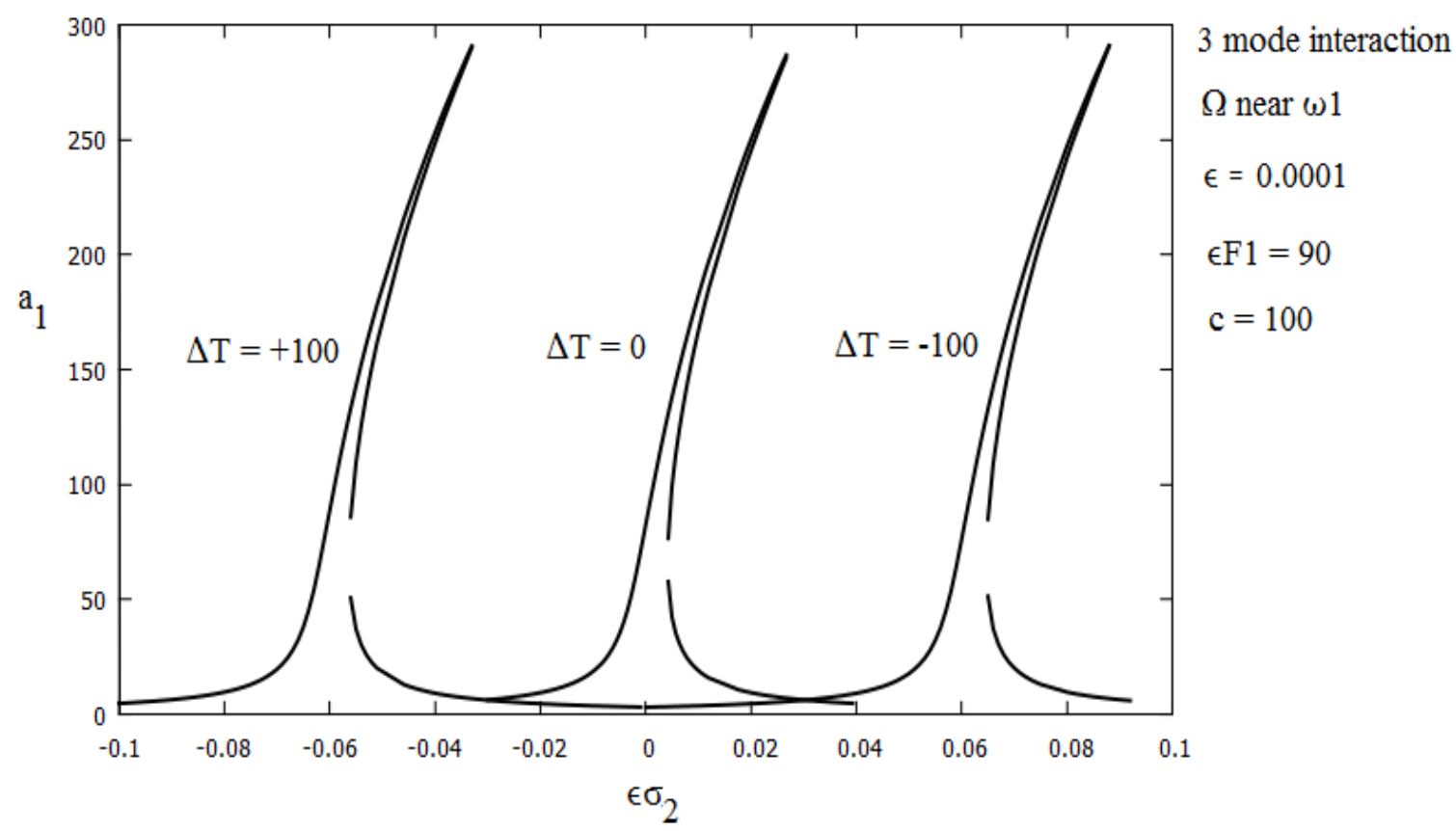

Fig. 8b. Variation of amplitude a1 of first mode with excitation frequency when the first mode is excited $\left(\Omega \approx \omega_{1}\right)$ at temperatures $\Delta \mathrm{T}=+100, \Delta \mathrm{T}=0$ and $\Delta \mathrm{T}=-100$ 


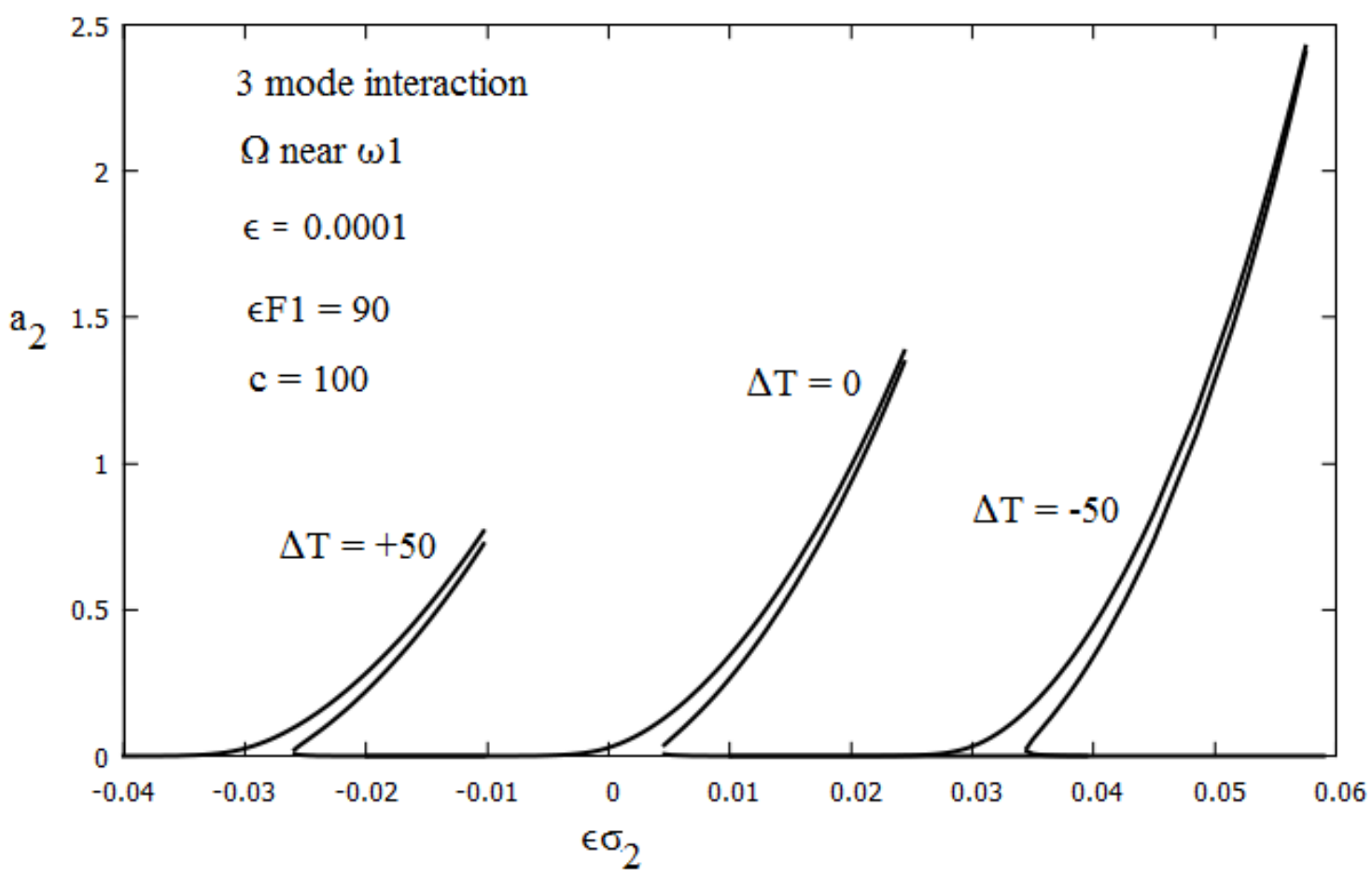

Fig. 9a. Variation of amplitude a2 of second mode with excitation frequency when the first mode is excited $\left(\Omega \approx \omega_{1}\right)$ at temperatures $\Delta \mathrm{T}=+50, \Delta \mathrm{T}=0$ and $\Delta \mathrm{T}=-50$

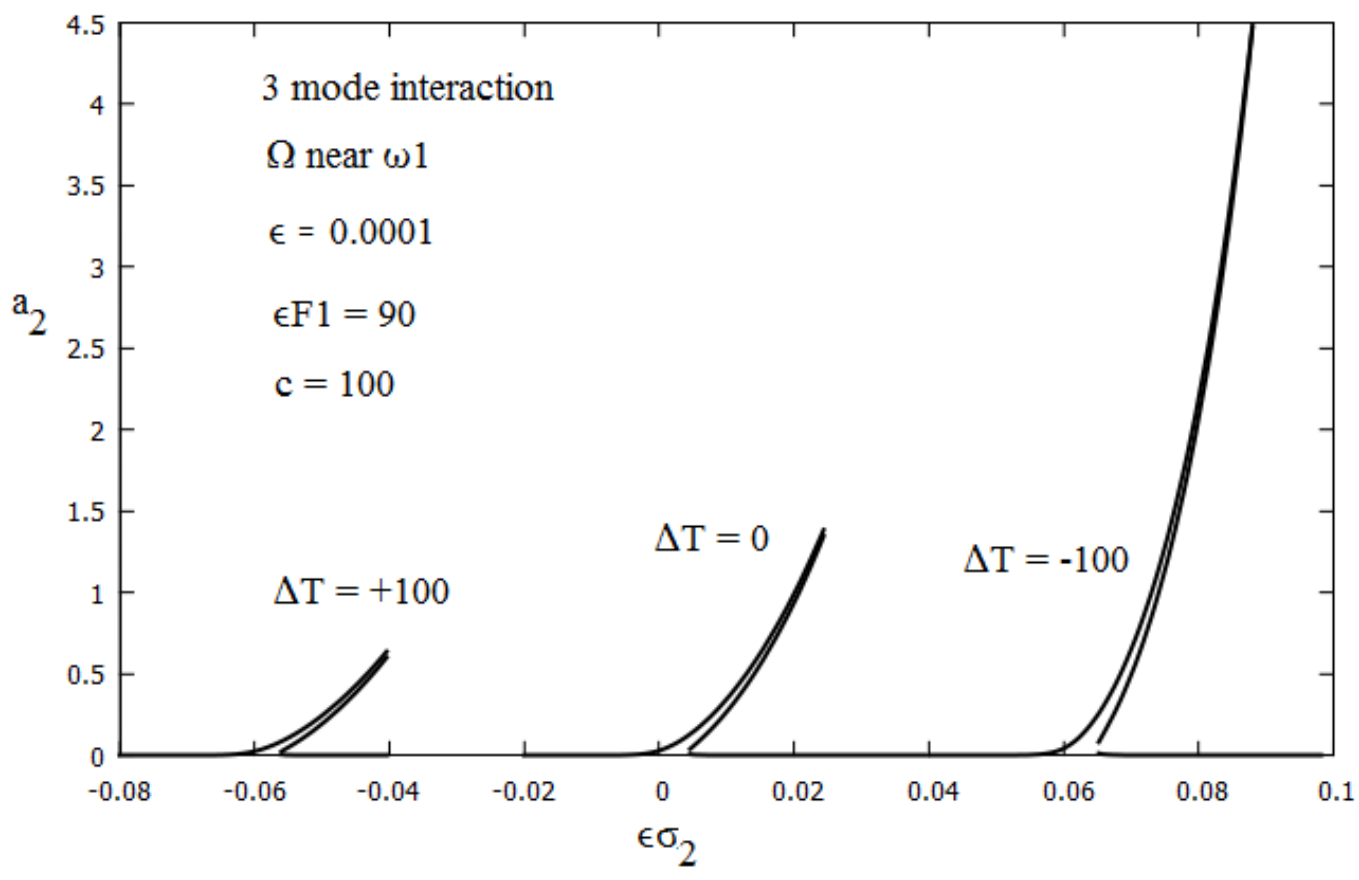

Fig. 9b. Variation of amplitude a2 of second mode with excitation frequency when the first mode is excited $\left(\Omega \approx \omega_{1}\right)$ at temperatures $\Delta \mathrm{T}=+100, \Delta \mathrm{T}=0$ and $\Delta \mathrm{T}=-100$ 


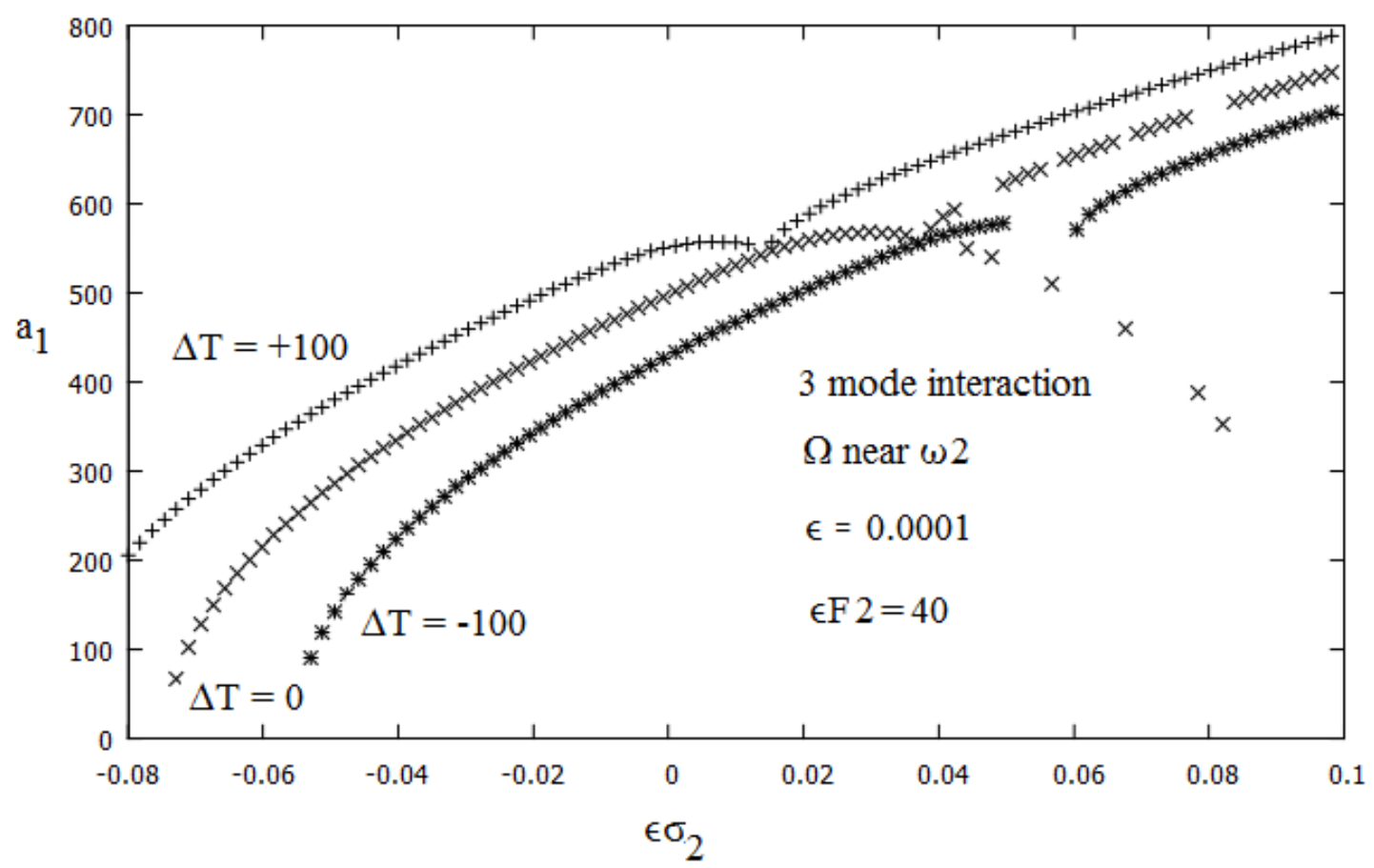

Fig. 10a. Variation of amplitude a1 of first mode with excitation frequency when the second mode is excited $\left(\Omega \approx \omega_{2}\right)$ at temperatures $\Delta \mathrm{T}=+100, \Delta \mathrm{T}=0$ and $\Delta \mathrm{T}=-100$

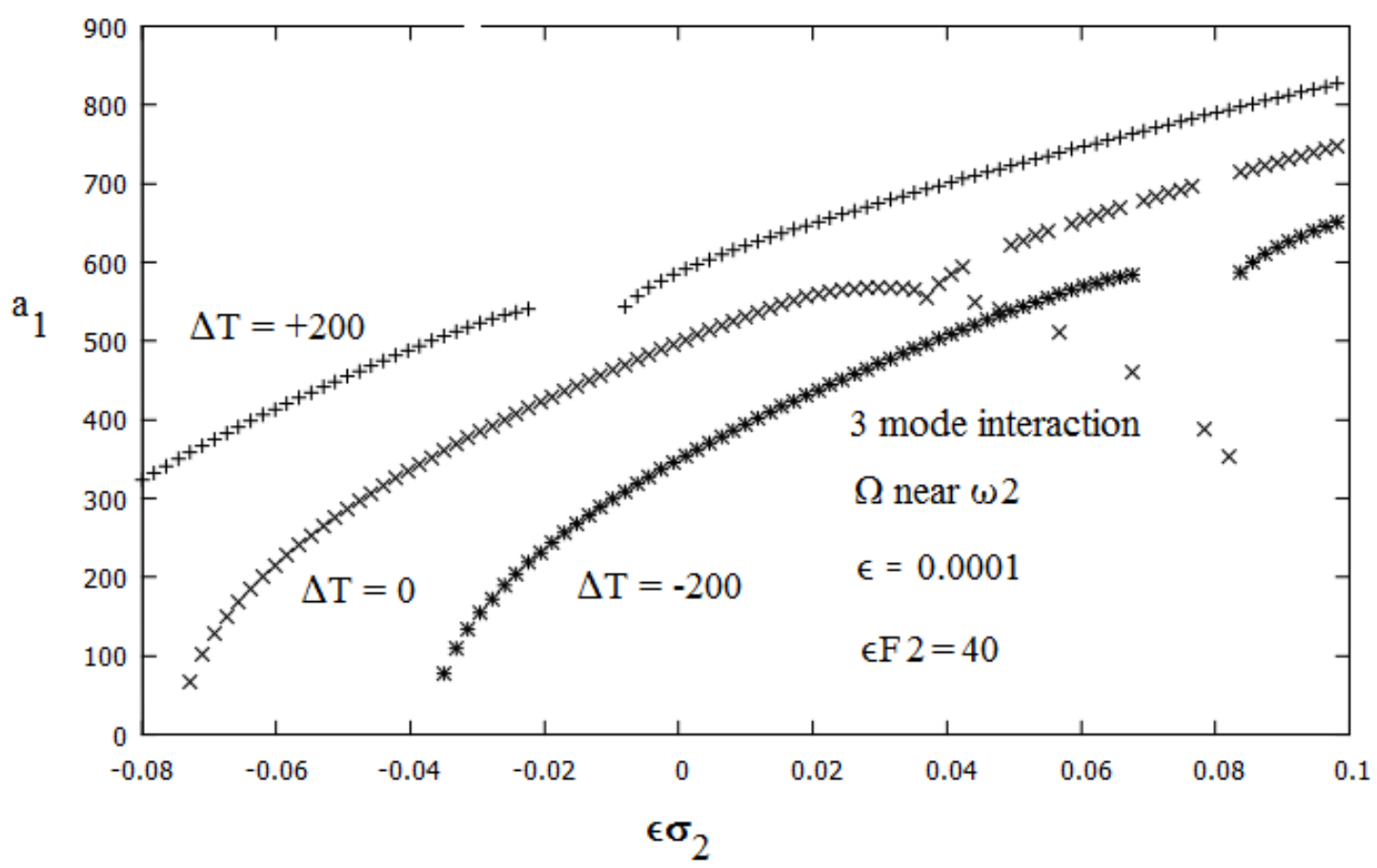

Fig. 10b. Variation of amplitude a1 of first mode with excitation frequency when the second mode is excited $\left(\Omega \approx \omega_{2}\right)$ at temperatures $\Delta \mathrm{T}=+200, \Delta \mathrm{T}=0$ and $\Delta \mathrm{T}=-200$ 


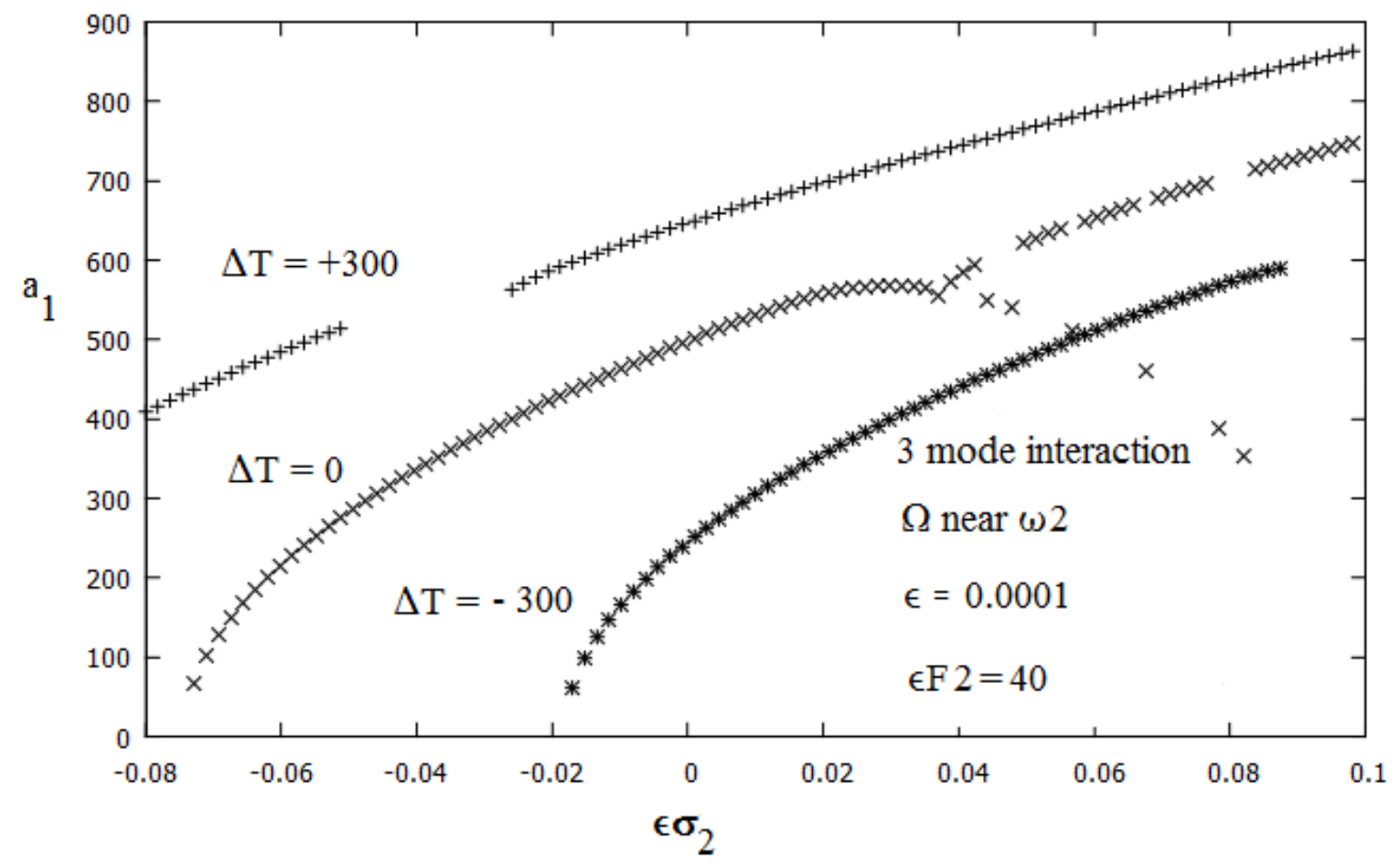

Fig. 10c. Variation of amplitude a1 of first mode with excitation frequency when the second mode is excited $\left(\Omega \approx \omega_{2}\right)$ at temperatures $\Delta \mathrm{T}=+300, \Delta \mathrm{T}=0$ and $\Delta \mathrm{T}=-300$

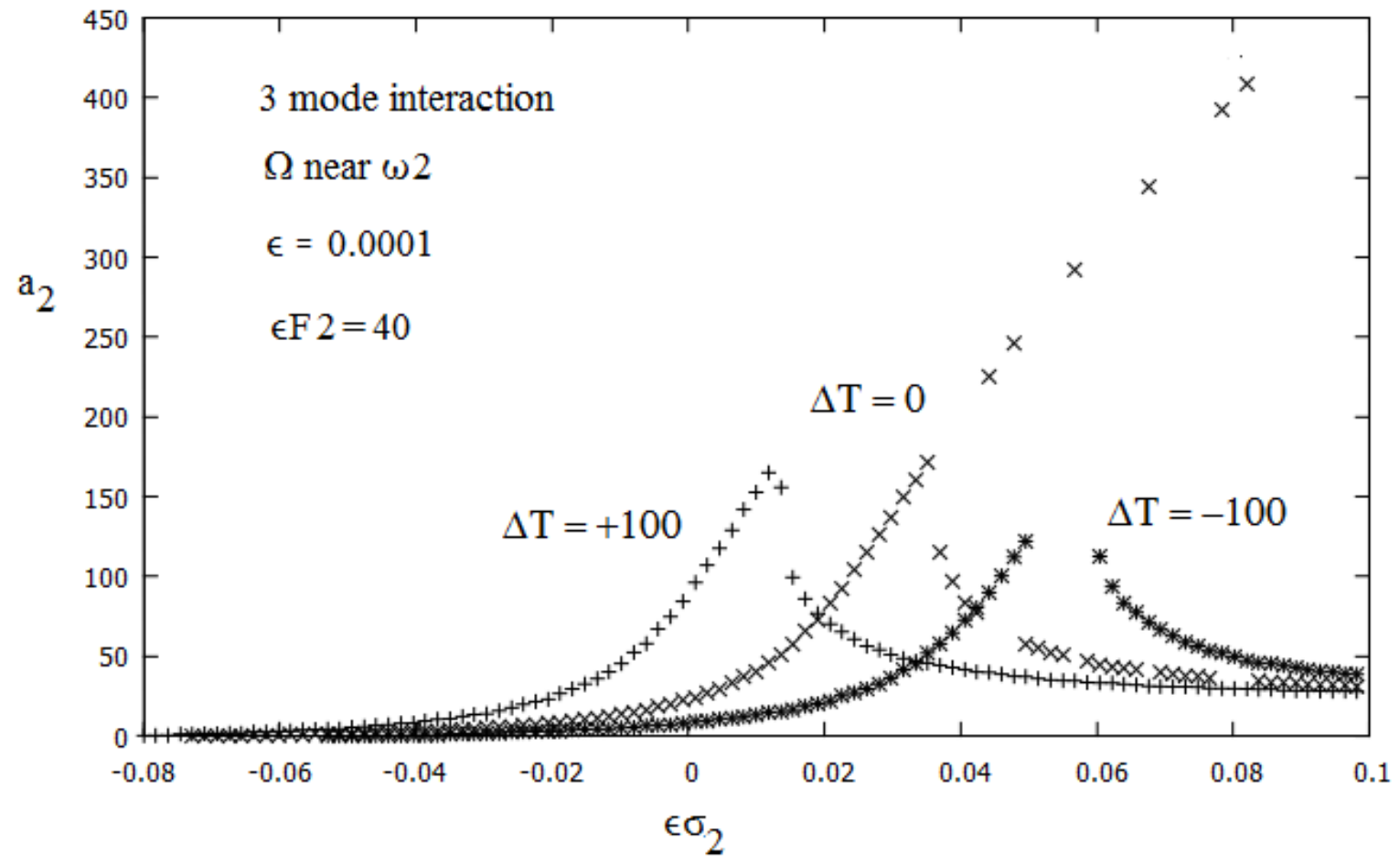

Fig. 11a. Variation of amplitude a 2 of second mode with excitation frequency when the second mode is excited $\left(\Omega \approx \omega_{2}\right)$ at temperatures $\Delta \mathrm{T}=+100, \Delta \mathrm{T}=0$ and $\Delta \mathrm{T}=-100$ 


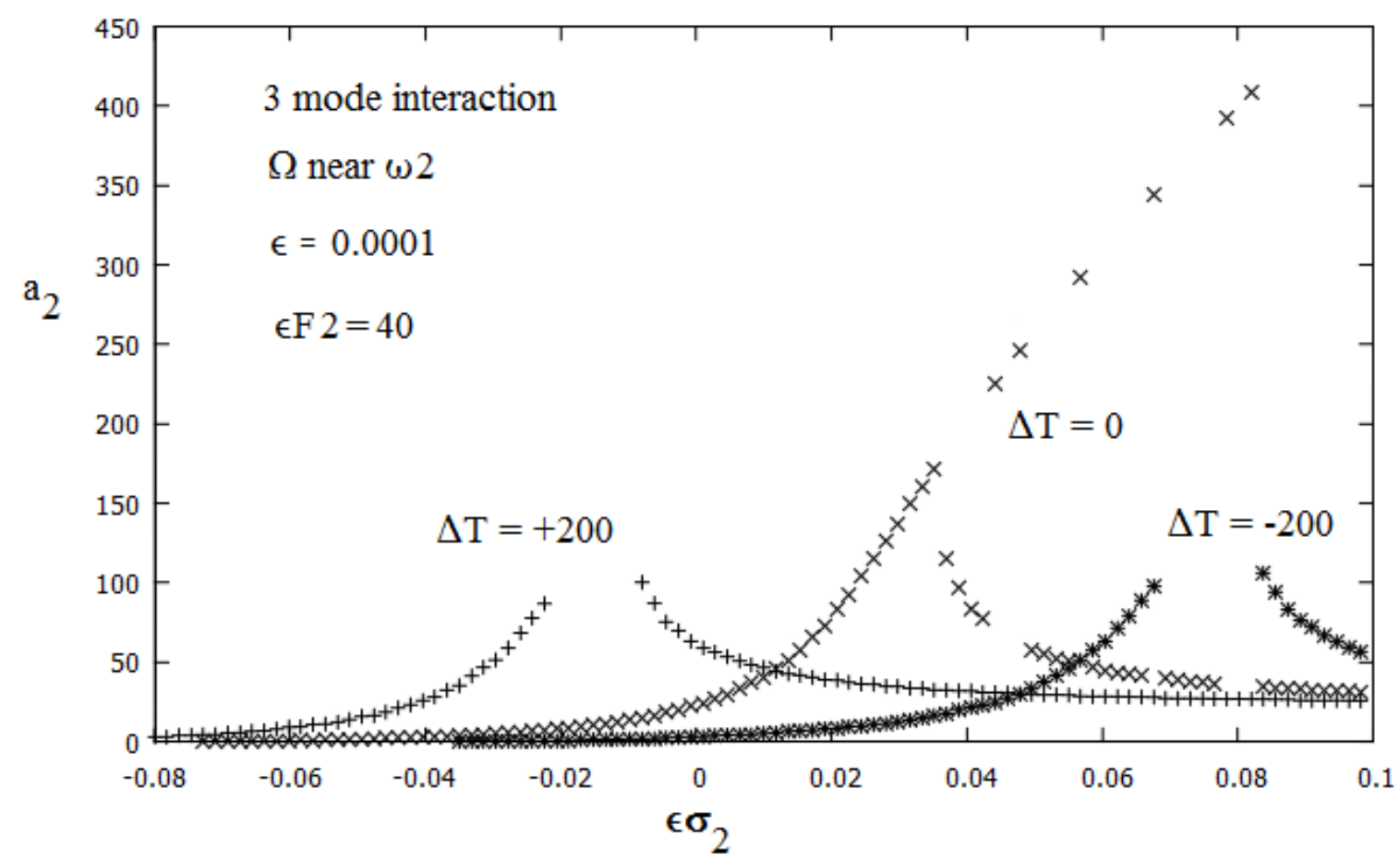

Fig. 11b. Variation of amplitude a 2 of second mode with excitation frequency when the second mode is excited $\left(\Omega \approx \omega_{2}\right)$ at temperatures $\Delta \mathrm{T}=+200, \Delta \mathrm{T}=0$ and $\Delta \mathrm{T}=-200$

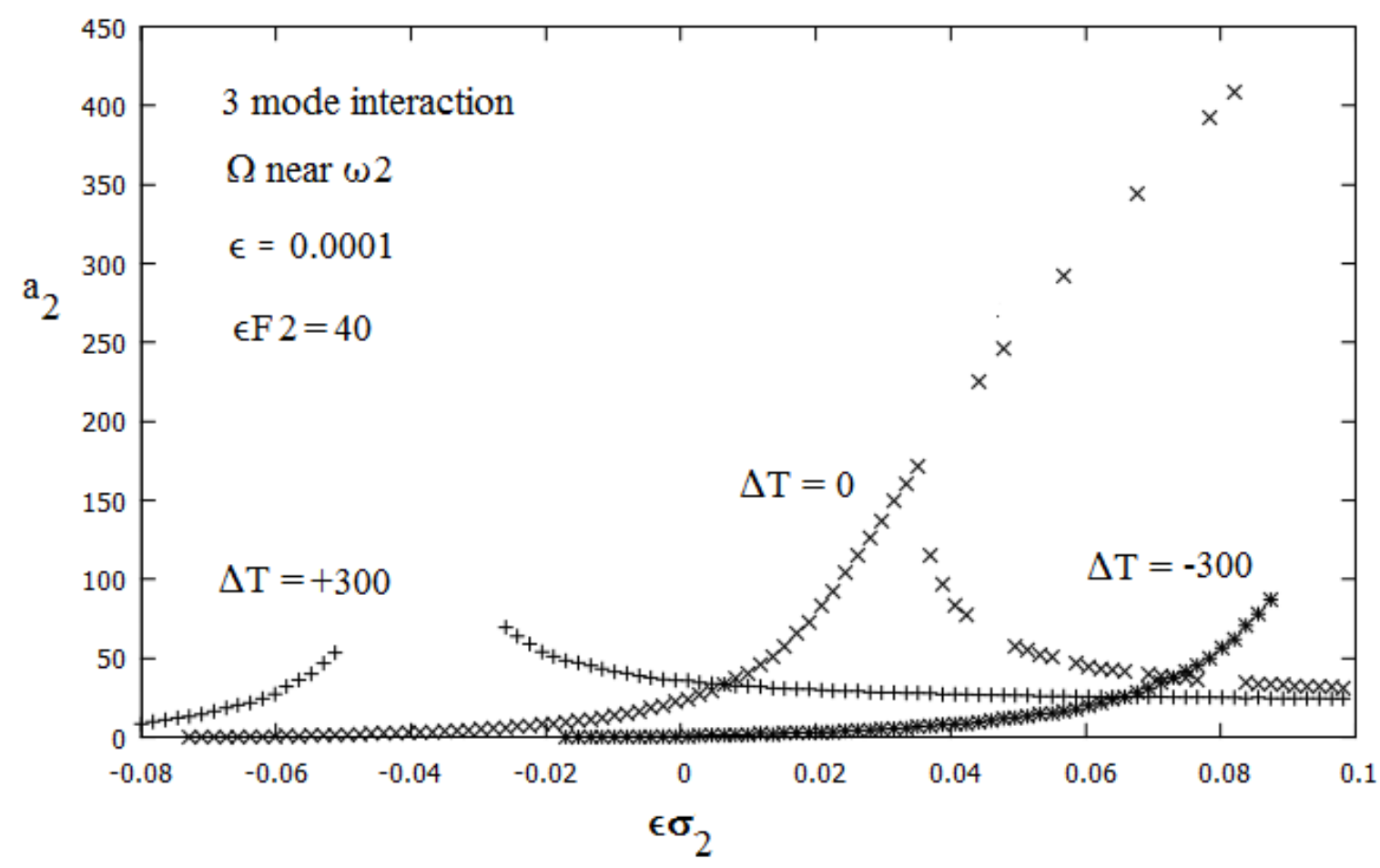

Fig. 11c. Variation of amplitude a 2 of second mode with excitation frequency when the second mode is excited $\left(\Omega \approx \omega_{2}\right)$ at temperatures $\Delta \mathrm{T}=+300, \Delta \mathrm{T}=0$ and $\Delta \mathrm{T}=-300$ 


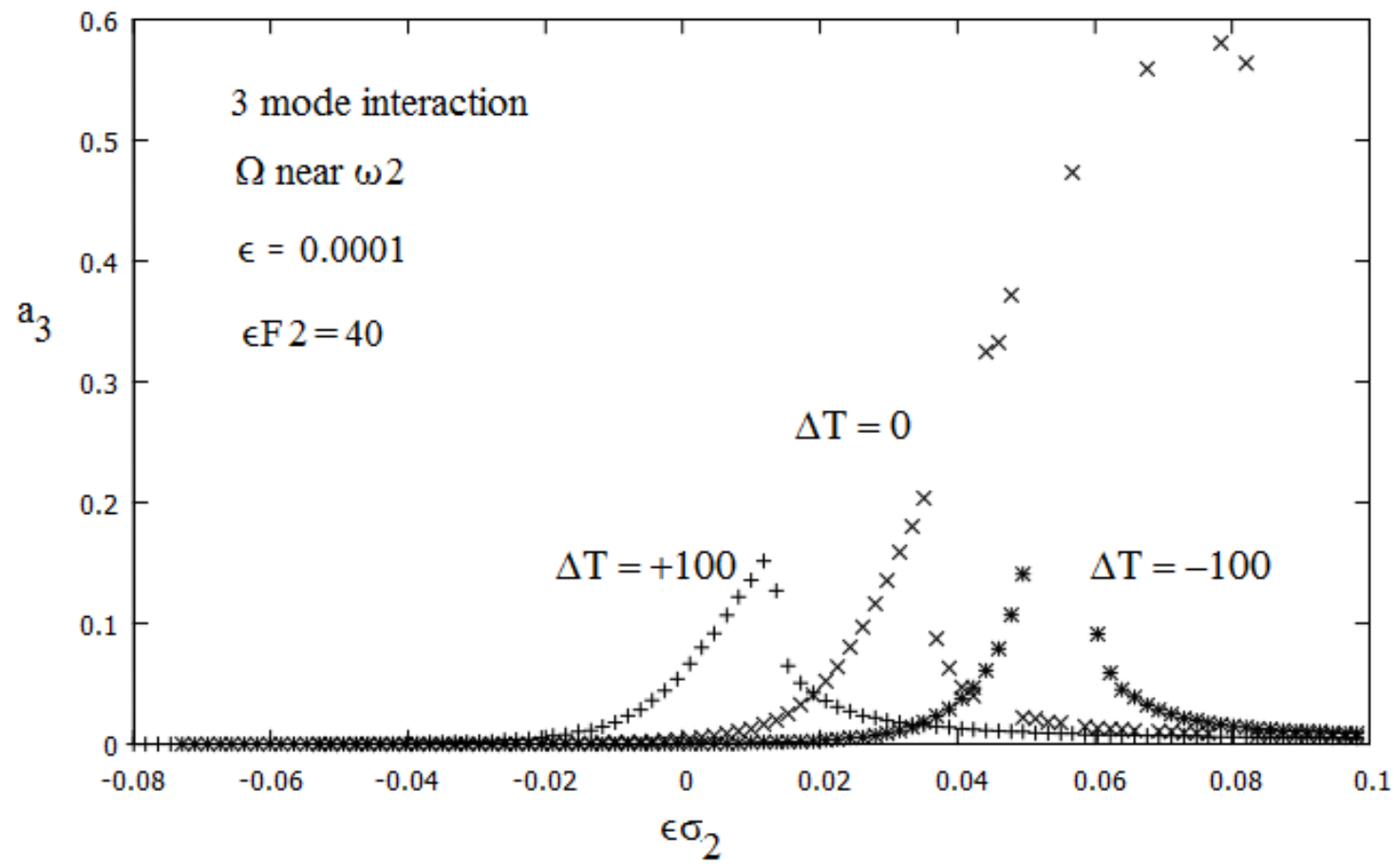

Fig. 12a. Variation of amplitude a3 of third mode with excitation frequency when the second mode is excited $\left(\Omega \approx \omega_{2}\right)$ at temperatures $\Delta \mathrm{T}=+100, \Delta \mathrm{T}=0$ and $\Delta \mathrm{T}=-100$

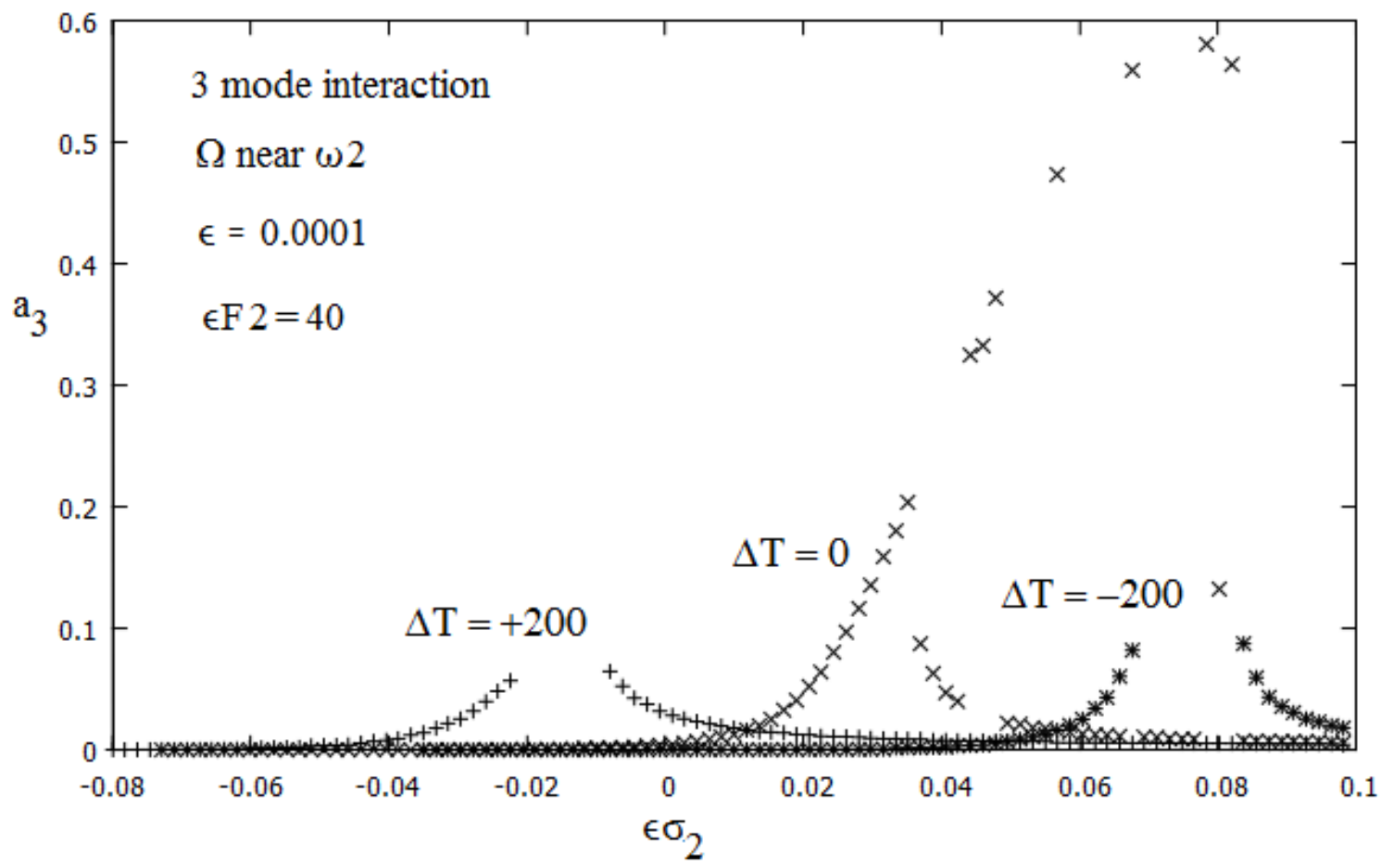

Fig. 12b. Variation of amplitude a3 of third mode with excitation frequency when the second mode is excited $\left(\Omega \approx \omega_{2}\right)$ at temperatures $\Delta \mathrm{T}=+200, \Delta \mathrm{T}=0$ and $\Delta \mathrm{T}=-200$ 


\section{Conclusion}

The vibrational behavior of a hinged-clamped beam with multimodal interaction under combined actions of periodic mechanical loadings and thermal loads has been investigated. Nonlinear PDEs governing the planar motion of the thermoelastic problem has been established and reduced to a set of nonlinear non-autonomous ODEs. For the sake of steadystate solutions, the ODEs have been reduced to three sets of parametric nonlinear algebraic equations solved by the mean of the Newton-Raphson technique. Results have been presented in terms of temperature effects on the response amplitudes of each of the three considered modes when one of them is directly excited. It came out that thermal effects are of great influence on the responses of such a system. More precisely, the temperature change affects the amplitude response curves by shifting them on either side of the temperature-free response curve in the frequency domain, depending on the sign and magnitude of the temperature difference. In terms of oscillation amplitude, the influence of temperature is diversely observed on the substructures depending on the magnitude and sign of the temperature difference but also on the mode directly excited. It is found that higher oscillation amplitude is observed for negative temperature difference for some substructures, while it is observed for positive one for some others and for temperature free-response curve for others. It should, however, be pointed out that for some substructures, temperature change does not significantly influence the oscillation amplitude. As a consequence, this study should contribute to advising civil and Structural designers that evaluating temperature influence on the structural elements engaged in vibrational behavior at a flat rate can produce inefficient results. The future work will be dedicated to the investigation of the dynamical aspect of the studied system in order to have more insights into the system responses to the combined effects of thermal loads and mechanical excitations in the presence of multimodal interaction.

\section{Declarations}

Conflicts of Interest: The authors declare no conflict of interest.

\section{References}

[1] S. A. Emam and A. H. Nayfeh, "Nonlinear responses of buckled beam to subharmonic-resonance excitations," Nonlinear Dynamics, vol. 35, pp 105-122, 2004. https://doi.org/10.1023/B:NODY.0000020878.34039.d4

[2] P. Ribeiro and R. Carneiro, "Experimental detection of modal interaction in the nonlinear vibration of a hinged-hinged beam," Journal of Sounds and Vibration, vol. 277, pp 943-954, 2004. https://doi.org/10.1016/j.jsv.2003.09.049

[3] S. A. Emam and A. H. Nayfeh, "Nonlinear response of buckled beams to 1:1 and 3:1 internal resonances," International Journal of Nonlinear Mechanics, vol. 52, pp 12-25, 2013. https://doi.org/10.1016/j.ijnonlinmec.2013.01.018

[4] S. A. Emam and M. M. Abdalla, "Subharmonic parametric resonance of simply supported buckled beams," Nonlinear Dynamics, vol. 79, pp 1443-1456, 2014. https://doi.org/10.1007/s11071014-1752-z

[5] D. S. Cho and W. K. Lee, "Modal interactions of a randomly excited hinged-clamped beam," Journal of Sound and Vibration, vol. 237, pp 377-393, 2000. https://doi.org/10.1006/jsvi.2000.3030

[6] A. H. Nayfeh and S. Sridhar, "Nonlinear analysis of the forced response of structural elements," Acoustical Society of America vol. 55, pp 281-291, 1974. https://doi.org/10.1121/1.1914499

[7] W. K. Lee and K. Y. Soh, "Nonlinear analysis of the forced responses of a beam with three mode interactions," Nonlinear Dynamics, vol. 6, pp 49-68, 1994. https://doi.org/10.1007/BF00045432

[8] W. K. Lee and C. H. Kim, "Evolution of domains of attraction of a forced beam with two mode interaction," Nonlinear Dynamics, vol. 14, pp 37-48, 1997. https://doi.org/10.1023/A:1008214107584 
[9] B. H. Lee and R. A. Ibrahim, "Stochastic bifurcation in non-linear structural systems near 1:1 internal resonance," Probabilistic Engineering Mechanics, vol. 9, pp 23-32, 1994. https://doi.org/10.1016/0266-8920(94)90026-4

[10] E. Manoach and P. Ribeiro, "Coupled, thermoelastic, large amplitude vibrations of Timoshenko beams," International Journal of Mechanical Sciences, vol. 46, pp 1589-1606, 2004. https://doi.org/10.1016/j.ijmecsci.2004.10.006

[11] P. Ribeiro and E. Manoach, "The effect of temperature on the large amplitude vibrations of curved beams," Journal of Sound and Vibration, vol. 285, pp 1093-1107, 2005. https://doi.org/10.1016/j.jsv.2004.09.010

[12] Y. Xia, B. Chen, S. Weng, Y. Q. Ni and Y. L. Xu, "Temperature effect on vibration properties of civil structures: a literature review and case studies," Journal of Civil Structural Health Monitoring, vol. 2, pp 29-46, 2012. https://doi.org/10.1007/s13349-011-0015-7

[13] Z. Yaobing and H. Chaohui, "Temperature effects on nonlinear vibration behaviors of EulerBernoulli beams with different boundary conditions," Shock and Vibration, vol. 6, pp1-11, 2018. https://doi.org/10.1155/2018/9834629

[14] E. Manoach and S. Doneva, "Coupled thermo-elastic large amplitude vibration of bi-material beams," Advanced Structured Materials, vol. 134, pp. 227-242, 2020. https://doi.org/10.1007/978-3-030-47491-1_13

[15] L. Liu and G. Kardomateas, "Nonlinear Vibration of composite plate to harmonic excitation with initial imperfection in thermal environments," Composites Structures, vol. 209, pp 401-423, 2019. http://doi.org/10.1016/j.compstruct.2018.10.101

[16] A. Warminska and E. Manoach, "Vibrations of composite beam under thermal and mechanical loadings," Procedia Engineering, vol. 144, pp 959-966, 2016. https://doi.org/10.1016/j.proeng.2016.05.123

[17] A. Warminska and E. Manoach, "Regular and chaotic oscillations of a Timoshenko beam subjected to mechanical and thermal loadings," Continuum Mechanics and Thermodynamics, vol. 27, pp 719-737, 2015. https://doi.org/10.1007/s00161-014-0381-6

[18] A. Warminska, J. Warminski, "Nonlinear dynamics of a reduced multimodal Timoshenko beam subjected to thermal and mechanical loadings," Meccanica, vol. 49, pp 1775-1793, 2014. http://.doi.org/10.1007/s11012-014-9891-3

[19] Y. Zhao, J. Peng and L. Chen, "Effects of temperature variations on nonlinear planar free and forced oscillations at primary resonances of suspended cables," Nonlinear Dynamics, vol. 89, pp 2815-2827, 2017. http://.doi.org/10.1007/s11071-017-3627-6

[20] Y. Zhao, C. Huang, L. Chen and J. Pen, "Nonlinear vibration behaviors of suspended cables under two-frequency excitation with temperature effects," Journal of Sound and Vibration, vol. 416, pp 279-294, 2018. https://doi.org/10.1016/j.jsv.2017.11.035

[21] G. Y. Wu, "The analysis of dynamic instability and vibration motions of a pinned beam with transverse magnetic fields and thermal loads," Journal of Sound and Vibration, vol. 284, pp 343360, 2005. http://doi.org/10.1016/j.jsv.2004.06.034

[22] G. Y. Wu, "The analysis of dynamic instability on the large amplitude vibrations of a beam with transverse magnetic fields and thermal loads," Journal of Sound and Vibration, vol. 302, pp 167177, 2007. https://doi.org/10.1016/j.jsv.2006.11.012

[23] S. Maleki and A. Maghsoudi-Barhmi, "Effects of concurrent earthquake and temperature loadings on cable-stayed bridges," International Journal of Structural Stability and Dynamics, vol. 16, 2016. https://doi.org/10.1142/S0219455415500200

[24] H. Farokhi and M. H. Ghayesh, "Thermo-mechanical dynamics of perfect and imperfect Timoshenko microbeams," International Journal of Engineering Science, vol. 91, pp 12-33, 2015. https://doi.org/10.1016/j.ijengsci.2015.02.005

[25] J. L. Huang, R.K.L. Su, Y.Y. Lee and S.H. Chen, "Nonlinear vibration of a curved beam under uniform base harmonic excitation with quadratic and cubic nonlinearities," Journal of Sound and Vibration, vol. 330, pp 5151-5164, 2011. https://doi.org/10.1016/j.ijengsci.2015.02.005

[26] A. H. Nayfeh and D. T. Mook, Nonlinear Oscillations, John Wiley \& Sons, New York, USA, 1979 
[27] A. H. Nayfeh and B. Balachandram, Applied Nonlinear Dynamics: Analytical, Computational and Experimental Methods, Wiley Series in Nonlinear Science, John Wiley \& Sons, 1995. https://doi.org/10.1002/9783527617548

[28] A. H. Nayfeh and P. F. Pai, Linear and Nonlinear Structural Mechanics, Wiley-Interscience, New York, USA, 2004. https://doi.org/10.1002/9783527617562

[29] A. Luongo and D. Zulli, Mathematical Models of Beams and Cables, John Wiley \& Sons, Inc. Hoboken, USA, 2013. https://doi.org/10.1002/9781118577554

[30] W. Lacarbonara, Nonlinear Structural Mechanics, Springer, Berlin, 2013. https://doi.org/10.1007/978-1-4419-1276-3 